\title{
Effect of diet and physical activity based interventions in pregnancy on gestational weight gain and pregnancy outcomes: meta-analysis of individual participant data from randomised trials
}

\author{
The International Weight Management in Pregnancy (i-WIP) Collaborative Group
}

Correspondence to: KS Khan

k.s.khan@qmul.ac.uk

Additional material is published online only. To view please visit the journal online.

Cite this as: BMJ 2017;358:j3119 http://dx.doi.org/10.1136/bmj.j3119

Accepted: 15 June 2017

\section{ABSTRACT}

Objective

To synthesise the evidence on the overall and differential effects of interventions based on diet and physical activity during pregnancy, primarily on gestational weight gain and maternal and offspring composite outcomes, according to women's body mass index, age, parity, ethnicity, and pre-existing medical condition; and secondarily on individual complications.

\section{DESIGN}

Systematic review and meta-analysis of individual participant data (IPD).

DATA SOURCES

Major electronic databases from inception to February 2017 without language restrictions.

ELIGIBILITY CRITERIA FOR SELECTING STUDIES

Randomised trials on diet and physical activity based interventions in pregnancy.

\section{DATA SYNTHESIS}

Statistical models accounted for clustering of participants within trials and heterogeneity across trials leading to summary mean differences or odds ratios with $95 \%$ confidence intervals for the effects overall, and in subgroups (interactions).

RESULTS

IPD were obtained from 36 randomised trials (12 526 women). Less weight gain occurred in the intervention group than control group (mean difference $-0.70 \mathrm{~kg}$, $95 \%$ confidence interval -0.92 to $-0.48 \mathrm{~kg}, \mathrm{I}^{2}=14.1 \%$; 33 studies, 9320 women). Although summary effect estimates favoured the intervention, the reductions

\section{WHAT IS ALREADY KNOWN ON THIS TOPIC}

Increased weight gain in pregnancy is associated with maternal and fetal complications

Interventions based on diet or physical activity or both in pregnancy minimise gestational weight gain

Interventions based on diet and physical activity may have a potential role in preventing adverse pregnancy outcomes

\section{WHAT THIS STUDY ADDS}

Diet and physical activity based interventions consistently reduce gestational weight gain across various subgroups of women categorised by age, parity, body mass index, ethnicity, and pre-existing medical condition

The reduction in odds of adverse maternal and offspring composite outcomes with diet and physical activity is not significant, and does not vary across various subgroups of women

Interventions significantly lower the odds of caesarean section and have no effect on offspring outcomes

in maternal (odds ratio $0.90,95 \%$ confidence interval 0.79 to $1.03, \mathrm{I}^{2}=26.7 \% ; 24$ studies, 8852 women) and offspring $\left(0.94,0.83\right.$ to $1.08, \mathrm{I}^{2}=0 \%$; 18 studies, 7981 women) composite outcomes were not statistically significant. No evidence was found of differential intervention effects across subgroups, for either gestational weight gain or composite outcomes. There was strong evidence that interventions reduced the odds of caesarean section $(0.91,0.83$ to 0.99 , $\mathrm{I}^{2}=0 \%$; 32 studies, 11410 women), but not for other individual complications in IPD meta-analysis. When IPD were supplemented with study level data from studies that did not provide IPD, the overall effect was similar, with stronger evidence of benefit for gestational diabetes $\left(0.76,0.65\right.$ to $0.89, \mathrm{I}^{2}=36.8 \%$; 59 studies, 16885 women).

\section{CONCLUSION}

Diet and physical activity based interventions during pregnancy reduce gestational weight gain and lower the odds of caesarean section. There is no evidence that effects differ across subgroups of women.

\section{Introduction}

Half of all women of childbearing age worldwide are overweight or obese..$^{1-3}$ Obesity and excessive gestational weight gain put mother and offspring at risk, both in pregnancy and in later life..$^{4-6}$ The resultant costs to the health service and society are considerable. ${ }^{78}$ Increasingly, healthcare organisations and research funding bodies prioritise research on interventions and strategies to reduce maternal weight related adverse outcomes in pregnancy. ${ }^{9-12}$

Syntheses of study level data on effects of diet and physical activity based interventions in pregnancy ${ }^{13}$ have shown an overall benefit on limiting gestational weight gain, but the findings varied for their protective effect on maternal and offspring outcomes. ${ }^{13} 14$ Importantly, the subgroups of women who may benefit the most from such interventions are not known. ${ }^{15}$ For this, primary studies do not have sufficient power, ${ }^{16} 17$ and meta-analyses of study level data are limited by the absence of published details of subgroup effects, ${ }^{18}$ and by potential ecological bias. ${ }^{19}$ These problems can be addressed by evidence synthesis using raw individual level data from relevant studies. ${ }^{20} 21$

We undertook an individual participant data (IPD) meta-analysis to assess the effects of diet and physical activity based interventions, primarily on gestational weight gain and on maternal and offspring composite outcomes, in subgroups defined by body mass index (BMI), age, parity, ethnicity, and pre-existing medical 
condition. Furthermore, we assessed the overall effects, and those of individual interventions (diet, physical activity, mixed), on critically important maternal and offspring complications. In addition to using IPD, we also assessed the impact of incorporating study level data from other studies not providing IPD.

\section{Methods}

The IPD meta-analysis was performed using a prespecified protocol (PROSPERO CRD42013003804) ${ }^{22}$ and was reported in line with recommendations of the Preferred Reporting Items for Systematic reviews and Meta-Analysis of Individual Participant Data (PRISMAIPD). ${ }^{23}$

\section{Literature search and study identification}

We searched the major electronic databases Medline, Embase, Cochrane Database of Systematic Reviews, Database of Abstracts of Reviews of Effects, Cochrane Central Register of Controlled Trials, and Health Technology Assessment Database from October 2013 to March 2015 to update our previous search in this topic for randomised trials on diet and physical activity based interventions in pregnancy. ${ }^{13}$ The search was further updated in January 2016 and February 2017 to identify new studies. We searched the internet by using general search engines, and contacted researchers in the specialty to identify relevant trials. There were no language restrictions. Web appendix 1 provides details of the search strategy.

Two independent researchers (ER and NM, AAM, or EM) selected studies in a two stage process. In the first stage, potential citations were identified. Next, we did a detailed evaluation of the full manuscripts of potential papers and selected articles that fulfilled the eligibility criteria. We included randomised trials that assessed the effects of interventions based on diet, physical activity, and mixed interventions in pregnancy, on maternal and offspring outcomes. We classified complex interventions on diet and physical activity, including those with behavioural change components, as mixed interventions. We excluded studies that only included women with gestational diabetes at baseline, involved animals, reported only non-clinical outcomes, and were published before 1990. The primary outcomes were gestational weight gain, a composite of maternal outcomes, and a composite of offspring outcomes. The secondary outcomes were individual maternal and offspring complications. The components of the composite outcomes were determined by a two round Delphi survey of researchers in this specialty, and were considered to be critically important to clinical practice. ${ }^{24}$ The maternal composite outcome included gestational diabetes mellitus, hypertensive disorders of pregnancy, preterm delivery, and caesarean section. The offspring composite outcome included stillbirth, small for gestational age fetus, large for gestational age fetus, and admission of the newborn to a neonatal intensive care unit.

We defined gestational weight gain as the difference between maternal weight at antenatal booking and the last weight measured before delivery. We accepted the primary authors' definition and reporting of gestational diabetes mellitus, pregnancy induced hypertension, pre-eclampsia, caesarean section, stillbirth, and admission to a neonatal intensive care unit. We defined preterm delivery as birth before 37 weeks of gestation, and small for gestational age and large for gestational age as babies with a birth weight below the 10th and at or over the 90th centiles, respectively, adjusted for mother's BMI, parity, and gestational age at delivery. ${ }^{25}$

Establishment of IPD collaborative network and database-We established the International Weight Management in Pregnancy IPD Collaborative Group by contacting researchers of eligible studies. ${ }^{26} \mathrm{~A}$ bespoke database was developed, and we requested collaborators for relevant data in any format. We sent three reminders when there was no response.

\section{Quality assessment of the included studies}

Two independent reviewers assessed the quality of the randomised trials using a risk of bias tool for sequence generation, allocation concealment, blinding, incomplete outcome data, selective outcome reporting, and other potential sources of bias. ${ }^{27}$ We considered a study to have a high risk of bias if it scored as such in at least one of the following domains: randomisation, allocation concealment, blinding of outcome assessment, or incomplete outcome data; all items should be scored as low risk for a study to be classified as low risk of bias.

\section{Data extraction and assessment of IPD integrity}

Two independent reviewers (ER and NM) undertook data extraction at study level for inclusion and exclusion criteria, the characteristics of the intervention, and the reported outcomes. We sought to obtain IPD from relevant studies published until July 2015, which was the endpoint for IPD acquisition, to allow sufficient time for data cleaning, standardisation, and amalgamation of datasets. We also extracted the published study level data for all relevant studies published until February 2017, including those published beyond the individual data acquisition timeline, and those for which IPD were not provided by study authors.

We obtained IPD for individual maternal characteristics that were determined a priori, such as BMI, age, parity, ethnicity, socioeconomic status, and pre-existing medical conditions. Continuous variables were kept continuous, but some were also categorised when considered to be clinically useful. These included categorisations based on BMI (normal 18.5-24.9 kg/m², overweight $25-29.9 \mathrm{~kg} / \mathrm{m}^{2}$, obese $\geq 30 \mathrm{~kg} / \mathrm{m}^{2}$ ) and age (cut-off 20 years). Mother's ethnicity was classified as white or non-white. We used the mother's educational status to indicate socioeconomic status: low status if the mother did not complete secondary education to A level, medium if she completed secondary education (A level equivalent), and high if she completed any further higher education. We defined pre-existing medical conditions as diabetes mellitus, early onset of gestational diabetes, or hypertension.

We considered participants to be adherent to the intervention based on the following criteria: 
completion of at least $70 \%$ of the intervention protocol, dataset provided information on adherence in a yes or no format, or participant was deemed to be adherent as per the study criteria. We performed range and consistency checks on all IPD and produced summary tables. The randomisation ratio, baseline characteristics, and method of analysis in the IPD dataset were compared with the published information. Any discrepancies, missing data, obvious errors, and inconsistencies between variables or outlying values were queried and rectified as necessary with input from the original authors.

\section{Data synthesis}

To obtain summary estimates (mean difference for gestational weight gain and odds ratios for binary outcomes) and 95\% confidence intervals for the intervention effects for each primary outcome we undertook a two stage IPD meta-analysis. ${ }^{21}$ We assessed the effects across all interventions overall and for individual interventions. A two stage IPD metaanalysis was used to obtain summary estimates of the subgroup effects (interactions) of interest, which compared differential effects of interventions across the primary outcomes. Additionally we evaluated whether there are any differential effects of interventions for individual complications, according to BMI (normal, overweight, obese). All analyses were designed to preserve the intention to treat principle.

The first stage of the two stage meta-analysis involved analysing the IPD in each trial separately, to account for the clustering of participants within trials, and to obtain the estimates of interest and their variances. For the cluster randomised trials, we included a random intercept for a unit of randomisation to account for this further clustering. For the outcome of gestational weight gain, we used analysis of covariance in each trial to regress the final weight value against the intervention while adjusting for baseline weight and centres in cluster randomised trials. For maternal and offspring outcomes, we used a logistic regression model for each trial separately, with the intervention as a covariate. We excluded women with confirmed glucose intolerance or a hypertensive disorder at baseline, as defined by the primary authors, in the analysis of composite adverse pregnancy outcomes. To assess potential intervention effect modifiers, we extended the aforementioned models to include interaction terms between participant level covariates and the intervention (ie, treatment-covariate interaction terms).

In the second stage, we pooled the derived effect estimates (ie, treatment effects or treatment-covariate interactions) across trials using a random effects model fitted using restricted maximum likelihood. The random effects approach allowed us to account for unexplained interstudy heterogeneity in effects across studies. This produced summary estimates and 95\% confidence intervals for the intervention effects and the interactions (subgroup effects). The Hartung-Knapp correction was applied when subsequently deriving 95\% confidence intervals for the true mean effect, to help account for the uncertainty of the estimate of interstudy heterogeneity. ${ }^{28} 29$

We included studies that did not contribute IPD, by incorporating their extracted study level data within the second stage of the IPD meta-analysis framework, to obtain summary estimates of intervention effects that combined IPD and non-IPD studies. Sensitivity analyses were also performed by excluding studies with high risk of bias, analysing the primary outcomes separately for each intervention type (diet, physical activity, and mixed), excluding participants not adherent to the intervention, by analysing change in BMI instead of weight gain, and excluding maternal weight gain estimates from pregnancies that ended before 37 completed weeks of gestation to avoid systematic differences.

Heterogeneity was summarised using the $\mathrm{I}^{2}$ statistic, the estimated interstudy variance $\left(\tau^{2}\right),{ }^{30}$ and approximate $95 \%$ prediction intervals, which indicate the potential intervention (or interaction) effect in a new population similar to those included in the metaanalysis. $^{31}$

Small study effects (potential publication bias) were investigated by using contour enhanced funnel plots alongside visual examination and statistical tests for asymmetry (Egger's test for continuous outcomes or Peter's test for binary outcomes). ${ }^{32}$ We assessed for IPD availability bias by comparing the summary results when including non-IPD studies with those from IPD studies. $^{33}$ Furthermore, we compared the symmetry of funnel plots before and after inclusion of non-IPD studies. All meta-analyses were undertaken using Stata software version 12.1 (StataCorp, College Station, TX, USA), and statistical significance was considered at the 5\% level.

\section{Patient involvement}

No patients were involved in setting the research question or the outcome measures, nor were they involved in developing plans for recruitment, design, or implementation of the study. A patient representative provided an input to the interpretation and writing up of results. There are no plans to disseminate the results of the research to study participants or the relevant patient community. It was not evaluated whether the studies included in the review had any patient involvement.

\section{Results}

\section{Study selection}

We identified 58 trials published up to June 2015, of which 36 studies (62\%) provided individual participant data (IPD), ${ }^{16} 17$ 34-66 that accounted for data from $80 \%$ of the participants (12 526/15 541); 22 studies (3015 women) did not provide IPD (fig 1)..$^{67-88}$ A further 45 trials (9945 women) ${ }^{89-133}$ were identified after the IPD acquisition timeline until February 2017.

\section{Characteristics of included studies and participants} IPD were available from 36 trials in 16 countries. Twenty two studies ${ }^{173436-394142474851-5356-6367}$ were 


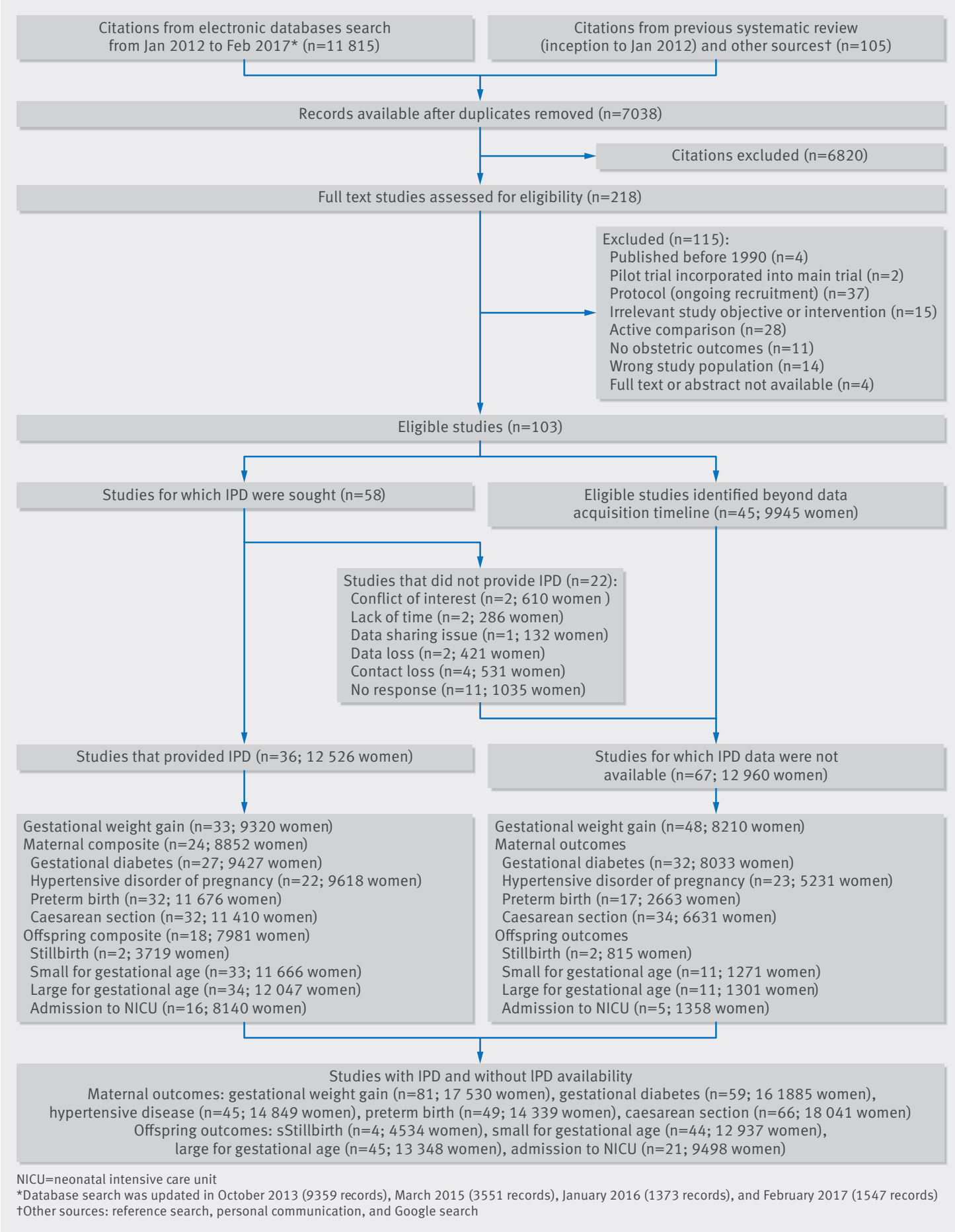

Fig 1 | Identification and selection of studies in individual participant data (IPD) meta-analysis of diet and physical activity based interventions on pregnancy outcomes after gestational weight gain

from Europe, four each from North America, ${ }^{44} 54$ 6566 Australia, ${ }^{16434550}$ and Brazil, ${ }^{35495564}$ and one study each from Egypt ${ }^{40}$ and Iran. ${ }^{46}$ Twenty three IPD studies included women of any body mass index (BMI), 34-38 42 44-48 52 54-56 58-61 64-67 seven included only obese women, ${ }^{17} 39-41506263$ and six included obese and overweight women. ${ }^{164349515357}$ The interventions included those mainly based on diet (four IPD studies) 47616264 or physical activity
(16 IPD studies), ${ }^{35-37424649-52555859656669}$ and those based on a mixed approach of diet, physical activity, or behaviour modifying techniques, or all three together (15 IPD studies). ${ }^{16} 17 \quad 34$ 39-41 $43-45 \quad 48$ 5354566063 One study had a three arm design with intervention arms being physical activity only and a mixed approach. ${ }^{57}$ The web appendix provides the characteristics of all IPD studies, and also those that did not contribute IPD. 


\begin{tabular}{|c|c|c|c|}
\hline Characteristics & No of studies (No of women) & Intervention & Control \\
\hline Mean (SD) age (years) & $35(12006)$ & $30.0(5.1)$ & $30.1(5.2)$ \\
\hline Weight (body mass index): & $34(12031)$ & & \\
\hline Normal $\left(18.5-24.9 \mathrm{~kg} / \mathrm{m}^{2}\right)$ & & $1974(31.7)$ & $1842(31.8)$ \\
\hline Overweight $\left(25-29.9 \mathrm{~kg} / \mathrm{m}^{2}\right)$ & & $1578(25.3)$ & $1523(26.3)$ \\
\hline Obese $\left(\geq 30 \mathrm{~kg} / \mathrm{m}^{2}\right)$ & & $2680(43.0)$ & $2434(42.0)$ \\
\hline Race/ethnicity: & $27(10020)$ & & \\
\hline White (including Russians and Australians) & & $4562(88.0)$ & $4217(87.2)$ \\
\hline Asian & & $157(3.0)$ & $156(3.2)$ \\
\hline Black & & $292(5.6)$ & $292(6.0)$ \\
\hline Central and South American & & $67(1.3)$ & $64(1.3)$ \\
\hline Middle Eastern (including Iranian and Turkish) & & $37(0.7)$ & $37(0.8)$ \\
\hline Other & & $71(1.4)$ & $68(1.4)$ \\
\hline Educational status of mothert: & $29(8914)$ & & \\
\hline Low & & $722(15.6)$ & $724(16.9)$ \\
\hline Medium & & $1372(29.6)$ & $1292(30.2)$ \\
\hline High & & $2536(54.8)$ & $2268(52.9)$ \\
\hline Smoker & $29(10958)$ & $875(15.4)$ & $865(16.4)$ \\
\hline Parity: & $33(11805)$ & & \\
\hline 0 & & $3027(49.5)$ & $2692(47.3)$ \\
\hline 1 & & $2136(34.9)$ & $2083(36.6)$ \\
\hline 2 & & $647(10.6)$ & $634(11.1)$ \\
\hline 3 & & $179(2.9)$ & $165(2.9)$ \\
\hline$\geq 4$ & & $129(2.1)$ & $113(2)$ \\
\hline No exercise or sedentary & $27(7583)$ & $1761(44.6)$ & $1731(47.6)$ \\
\hline Pre-existing diabetes mellitus & $25(9589)$ & $6(0.1)$ & $9(0.2)$ \\
\hline Pre-existing hypertension & $23(5494)$ & $73(2.5)$ & $54(2.1)$ \\
\hline
\end{tabular}

*Proportion out of observations in control or intervention arms, respectively.

tLow=not completed secondary education to A level; medium=completed secondary education (A level equivalent); high=any further or higher education.

More than $80 \%$ of women in the IPD meta-analyses were of white origin, and at least half were classified as of high socioeconomic status. Around $45 \%$ of women were nulliparous, $40 \%$ were obese, and a similar proportion was classified as having sedentary status with no exercise at baseline (table 1). IPD were available to assess the effects of interventions on gestational weight gain (33 studies, 9320 women), maternal composite outcomes (24 studies,

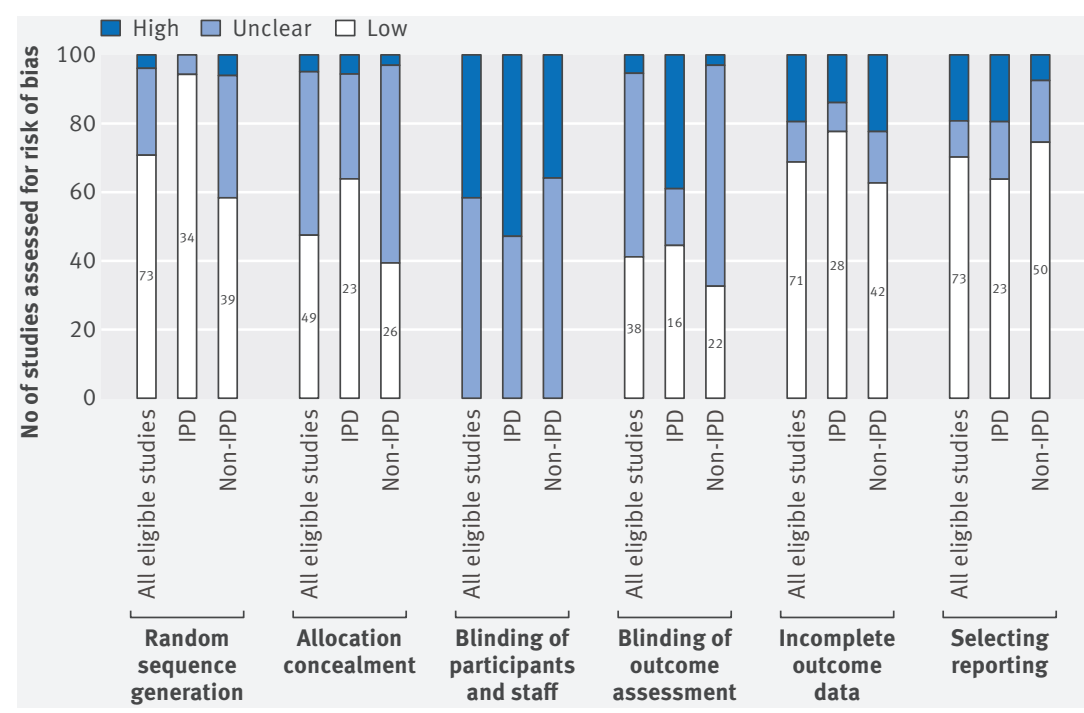

Fig 2 | Assessment of risk of bias in all eligible studies ( $n=103)$, studies with individual participant data (IPD) $(n=36)$, and studies without access to IPD $(n=67)$
8852 women), and offspring composite outcomes (18 studies, 7981 women). The largest IPD were available for the outcome of large for gestational age fetus (34 studies, 12047 women), followed by preterm delivery (32 studies, 11676 women), small for gestational age fetus (33 studies, 11666 women), any caesarean section (32 studies, 11410 women), hypertensive disorders of pregnancy (22 studies, 9618 women), and gestational diabetes (27 studies, 9427 women). We did not have access to IPD for $51 \%$ of all eligible women $(12960 / 25486)$ from 67 studies (fig 1).

\section{Quality of included studies}

Overall, trials had a low risk of bias in random sequence generation $(71 \%, 73 / 103)$. More than $90 \%$ (34/36) of studies that contributed to the IPD were assessed as low risk of bias in this domain compared with $58 \%(28 / 67)$ of the non-IPD studies. Two IPD studies (2/36) and one non-IPD study (3/67) were considered high risk for allocation concealment. Blinding of outcome assessment was appropriate in 44\% (16/36) of IPD and 33\% (22/67) of non-IPD studies (fig 2). Fewer IPD studies (5/36) were assessed as high risk of bias for incomplete outcome data than non-IPD studies (15/67). Figure 2 shows the summary of the risk of bias estimates for all eligible studies and those that did and did not contribute to IPD. We did not encounter any issues that we were not able to clarify with the IPD contributor during the IPD integrity check. 


\begin{tabular}{|c|c|c|c|c|c|c|c|c|c|c|}
\hline \multirow[b]{2}{*}{ Outcomes } & \multicolumn{2}{|c|}{ No of studies (No of women) } & \multicolumn{2}{|c|}{ Intervention } & \multicolumn{2}{|l|}{ Control } & \multicolumn{2}{|l|}{ Mean difference $(95 \% \mathrm{Cl})$} & \multicolumn{2}{|c|}{$I^{2}(\%)$} \\
\hline & IPD & IPD and non-IPD & IPD & $\begin{array}{l}\text { IPD and } \\
\text { non-IPD }\end{array}$ & IPD & $\begin{array}{l}\text { IPD and } \\
\text { non-IPD }\end{array}$ & IPD & IPD and non-IPD & IPD & $\begin{array}{l}\text { IPD and } \\
\text { non-IPD }\end{array}$ \\
\hline Overall & $33(9320)$ & $81(17530)$ & $10.1(5.4)$ & $10.6^{*}$ & $10.8(5.4)$ & $11.5^{\star}$ & $-0.70(-0.92$ to -0.48$)$ & $-1.10(-1.46$ to -0.74$)$ & 14.1 & 73.8 \\
\hline Diet & $4(1168)$ & $12(2017)$ & $10.2(4.4)$ & $9.2^{*}$ & $11.0(4.8)$ & $11.7^{\star}$ & $-0.72(-1.48$ to 0.04$)$ & $-2.84(-4.77$ to -0.91$)$ & 0.0 & 92.3 \\
\hline Physical activity & $15(2915)$ & $37(7355)$ & $9.8(4.4)$ & $11.3^{\star}$ & $10.8(4.8)$ & $11.9^{*}$ & $-0.73(-1.11$ to -0.34$)$ & $-0.72(-1.04$ to -0.41$)$ & 0.0 & 45.4 \\
\hline Mixed approach & $15(5369)$ & $35(8448)$ & $10.2(6.0)$ & $10.3^{*}$ & $10.6(5.9)$ & $11.0^{\star}$ & $-0.71(-1.10$ to -0.31$)$ & $-1.00(-1.39$ to -0.61$)$ & 34.9 & 54.6 \\
\hline
\end{tabular}

Effects of interventions on pregnancy outcomes Gestational weight gain

Based on IPD meta-analysis (33 studies, 9320 women), diet and physical activity based interventions resulted in significantly less gestational weight gain compared with control (summary mean difference $-0.70 \mathrm{~kg}, 95 \%$ confidence interval -0.92 to $-0.48 \mathrm{~kg}, \mathrm{I}^{2}=14.1 \%$ ), after adjusting for baseline weight and clustering. The approximate $95 \%$ prediction interval for the intervention effect in a new setting was -1.24 to $-0.16 \mathrm{~kg}$ (table 2).

\section{Differential effects in subgroups}

No strong evidence was found of a treatment-covariate interaction for baseline BMI when treated as a continuous covariate $(-0.02 \mathrm{~kg}$ change in intervention effect per one unit increase in BMI, 95\% confidence interval -0.08 to $0.04 \mathrm{~kg}$ ), or when compared as overweight versus normal $(-0.11 \mathrm{~kg},-0.77$ to $0.55 \mathrm{~kg})$, obese versus normal $(0.06 \mathrm{~kg},-0.90$ to $1.01 \mathrm{~kg})$, and obese versus overweight $(-0.09 \mathrm{~kg},-1.05$ to $0.86 \mathrm{~kg})$. We also did not observe evidence of a subgroup effect for age $(-0.03 \mathrm{~kg}$ per one year increase in age, $95 \%$ confidence interval -0.08 to $0.02 \mathrm{~kg})$, parity $(0.10 \mathrm{~kg}$ change in effect for multiparity versus nulliparity, 95\% confidence interval -0.39 to $0.60 \mathrm{~kg}$ ), ethnicity ( 0.05 kg change in effect for non-white versus white, $95 \%$ confidence interval -1.27 to $1.37 \mathrm{~kg}$ ), and underlying medical condition $(1.51 \mathrm{~kg}$ change in effect for women with at least one condition versus none, 95\% confidence interval -2.01 to $5.02 \mathrm{~kg}$ ). The findings were consistent when continuous covariates were analysed as categorical measures based on clinically relevant cut points (table 3).

\section{Sensitivity analyses}

The reduction in gestational weight gain owing to the intervention was consistently observed when the analysis was restricted to studies with low risk of bias $(-0.67 \mathrm{~kg}$, $95 \%$ confidence interval -0.95 to $-0.38 \mathrm{~kg}$; 15 studies, 5585 women), women adherent to the intervention ( $-0.76 \mathrm{~kg},-1.00$ to $-0.52 \mathrm{~kg}$; 33 studies, 8565 women), women followed up until more than 37 weeks' gestation ( $-0.91 \mathrm{~kg},-1.17$ to $-0.66 \mathrm{~kg} ; 28$ studies, 5324 women), and for BMI instead of maternal weight as an outcome $\left(-0.30 \mathrm{~kg} / \mathrm{m}^{2},-0.39\right.$ to $-0.21 \mathrm{~kg} / \mathrm{m}^{2} ; 31$ studies, 9238 women).

\begin{tabular}{|c|c|c|c|c|}
\hline \multirow[b]{2}{*}{ Maternal characteristics } & \multirow{2}{*}{$\begin{array}{l}\text { No of studies } \\
\text { (No of women) }\end{array}$} & \multirow{2}{*}{$\begin{array}{l}\text { Mean difference* } \mathrm{kg} \\
(95 \% \mathrm{Cl})\end{array}$} & \multicolumn{2}{|l|}{ Treatment covariate interaction } \\
\hline & & & Coefficient; 95\% Cl (95\% PI) & $I^{2}(\%)$ \\
\hline \multicolumn{5}{|l|}{ Baseline body mass index: } \\
\hline Normal & $21(3376)$ & $-0.77(-1.15$ to -0.39$)$ & \multirow[t]{3}{*}{$-0.02 ;-0.08$ to $0.04(-0.21$ to 0.17$) \dagger$} & \multirow[t]{3}{*}{39.8} \\
\hline Overweight & $28(2574)$ & $-0.75(-1.22$ to -0.27$)$ & & \\
\hline Obese & $31(3335)$ & $-0.85(-1.41$ to -0.29$)$ & & \\
\hline \multicolumn{5}{|l|}{ Parity: } \\
\hline Nulliparous & $27(4513)$ & $-0.80(-1.17$ to -0.43$)$ & \multirow[t]{2}{*}{$0.10 ;-0.39$ to $0.60(-0.83$ to 1.04$) \neq$} & \multirow[t]{2}{*}{4.8} \\
\hline Multiparous & $27(4548)$ & $-0.62(-0.88$ to -0.37$)$ & & \\
\hline \multicolumn{5}{|l|}{ Ethnicity: } \\
\hline White & $21(6814)$ & $-0.74(-1.07$ to -0.42$)$ & \multirow[t]{2}{*}{$0.05 ;-1.27$ to 1.37 ( -1.28 to 1.39$) \S$} & \multirow[t]{2}{*}{26.1} \\
\hline Non-white & $15(621)$ & $-0.42(-1.12$ to 0.28$)$ & & \\
\hline \multicolumn{5}{|l|}{ Age (years): } \\
\hline$\geq 20$ & $32(9045)$ & $-0.72(-0.95$ to -0.50$)$ & \multirow[t]{2}{*}{$-0.03 ;-0.08$ to 0.02 (-0.14 to 0.09$)$ व } & \multirow[t]{2}{*}{25.9} \\
\hline$<20$ & $13(232)$ & $0.05(-1.34$ to 1.44$)$ & & \\
\hline \multicolumn{5}{|c|}{ Pre-existing medical conditions ${ }^{\star \star}$ : } \\
\hline None & $18(4335)$ & $-0.62(-0.90$ to -0.34$)$ & \multirow[t]{2}{*}{$1.51 ;-2.01$ to $5.02(-4.13$ to 7.15$)+t$} & \multirow[t]{2}{*}{28.4} \\
\hline$\geq 1$ & $6(128)$ & $0.40(-1.92$ to 2.71$)$ & & \\
\hline \multicolumn{5}{|c|}{ 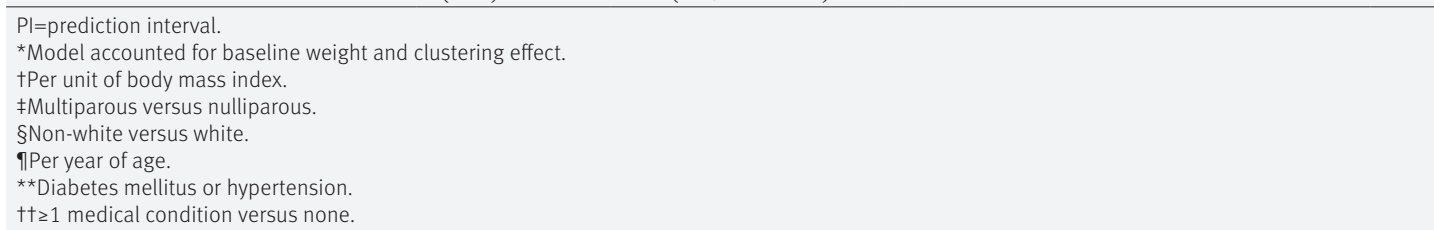 } \\
\hline
\end{tabular}


Addition of studies that did not contribute IPD

In meta-analysis undertaken by supplementing the IPD with study level data from studies (48 studies, 8210 women) that did not contribute IPD, we observed a larger beneficial intervention effect for weight gain (summary mean difference $-1.1 \mathrm{~kg}$; 95\% confidence interval -1.46 to $-0.74 \mathrm{~kg} ; 81$ studies, 17530 women). The benefit was also consistently observed for individual interventions based on diet, physical activity, or mixed approach (table 2).

\section{Maternal and offspring composite outcomes}

In the IPD meta-analyses, the summary estimates favoured the intervention group for reduction in odds of maternal (odds ratio 0.90, 95\% confidence interval 0.79 to $1.03, \mathrm{I}^{2}=26.7 \%$; 24 studies, 8851 women) and offspring composite outcomes $(0.94,0.83$ to 1.08 , $\mathrm{I}^{2}=0 \%$; 18 studies, 7981 women), but these were not statistically significant (table 4).

\section{Differential effects across subgroups}

We observed no strong evidence of differential subgroup effects for maternal composite outcome according to either baseline BMI (treatment-covariate interaction $1.00,95 \%$ confidence interval 0.98 to $1.02)$, age $(1.01,0.99$ to 1.03$)$, parity $(1.03,0.75$ to 1.39), ethnicity $(0.93,0.63$ to 1.37$)$, and underlying medical condition (1.44, 0.15 to 13.74 ) (table 5).

A similar lack of differential effect was observed for offspring composite outcome in mothers grouped according to baseline BMI (interaction 0.98, 95\% confidence interval 0.95 to 1.00$)$, age (1.01, 0.98 to $1.04)$, parity (0.94, 0.64 to 1.37$)$, ethnicity $(1.12,0.75$ to 1.68$)$, and underlying medical condition (0.58, 0.03 to 9.81 ) (table 4). The findings did not change for maternal and offspring composite outcomes when BMI and age were analysed as continuous instead of categorical variables.

\section{Individual maternal outcomes}

Overall, in the IPD meta-analysis we observed a significant reduction in caesarean section (odds ratio $0.91,95 \%$ confidence interval 0.83 to 0.99 , $\mathrm{I}^{2}=0 \%$; 32 studies, 11410 women) for interventions compared with routine care. The reduction in other individual outcomes such as gestational diabetes $\left(0.89,0.72\right.$ to $1.10, \mathrm{I}^{2}=23.8 \% ; 27$ studies, 9427 women), hypertensive disorders of pregnancy (0.95, 0.78 to $1.16, \mathrm{I}^{2}=24.2 \%$; 22 studies, 9618 women), and preterm delivery $\left(0.94,0.78\right.$ to $1.13, \mathrm{I}^{2}=17.3 \%$; 32 studies, 11676 women) were not statistically significant in IPD meta-analyses (table 5). We did not observe any differential effect according to baseline BMI category (normal, overweight, obese) for any of the individual maternal outcomes (see web appendix 3). The findings were consistent when study level data from non-IPD studies were meta-analysed with IPD, but with a stronger evidence of benefit for gestational diabetes. The reduction in gestational diabetes $(0.76$, 0.65 to $0.89,36.8 \%$; 59 studies, 16885 women) became significant (table 5).
Among individual interventions, those based mainly on physical activity showed a reduction in gestational diabetes in both IPD (odds ratio $0.67,95 \%$ confidence interval 0.46 to $0.99, \mathrm{I}^{2}=0 \%$; 10 studies, 2700 women) and in combined (IPD and non-IPD) meta-analyses (0.66, 0.53 to $0.83, \mathrm{I}^{2}=0 \%$; 27 studies, 6755 women). While the summary estimates for physical activity based interventions favoured caesarean section $(0.82$, 0.67 to $1.01, \mathrm{I}^{2}=0 \%$; 13 studies, 3046 women) and hypertensive disorders of pregnancy $(0.74,0.42$ to $1.33, \mathrm{I}^{2}=6.0 \%$; 7 studies, 2565 women) in IPD metaanalyses, the addition of non-IPD studies resulted in stronger evidence of benefit for these complications, with reduction in the respective odds by $17 \%(0.83$, 0.73 to $0.95, \mathrm{I}^{2}=0 \%$; 32 studies, 6587 women) and $32 \%\left(0.68,0.49\right.$ to $0.93, \mathrm{I}^{2}=0 \%$; 20 studies, 5125 women).

A strong effect was observed for preterm birth with diet based interventions in both IPD (odds ratio 0.28 , $95 \%$ confidence interval 0.08 to $0.96, \mathrm{I}^{2}=0 \%$; 4 studies, 1344 women) and combined analyses (0.32, 0.14 to $0.70, \mathrm{I}^{2}=0 \%$; 7 studies, 1696 women), but the overall sample sizes were relatively small (table 5). There was no evidence of benefit with mixed interventions for any maternal outcomes.

\section{Individual offspring outcomes}

No strong evidence was found that interventions had an effect on individual offspring outcomes such as stillbirth (odds ratio 0.81, 95\% confidence interval $<0.001$ to $256.69, \mathrm{I}^{2}=0 \%$; 2 studies, 3719 women), small for gestational age fetus (1.06, 0.94 to $1.20, \mathrm{I}^{2}=0 \%$; 33 studies, 11666 women), large for gestational age fetus $\left(0.90,0.76\right.$ to $1.07, \mathrm{I}^{2}=38.0 \%$; 34 studies, 12047 women), and admission to a neonatal intensive care unit $(1.01,0.84$ to 1.23 , $\mathrm{I}^{2}=0 \%$; 16 studies, 8140 women) based on the IPD meta-analyses. The significance of the findings did not change when non-IPD studies were added to the IPD meta-analyses (table 5). The numbers of eligible participants for whom data were obtained, effect estimates, and confidence intervals for all above analyses are available from the study authors on request. There was no differential effect for any individual offspring outcome according to the BMI category (see web appendix 3).

\section{Small study effects}

We found visual and statistical evidence (Egger's test $\mathrm{P}=0.04)$ of small study effects in the contour enhanced funnel plots for the IPD meta-analysis of the overall effect on gestational weight gain. The asymmetry of the plot was not improved by the addition of study level data from non-IPD studies to the meta-analysis. When studies with high risk of bias were excluded from the analysis, the symmetry of the funnel plot improved (Egger's test $\mathrm{P}=0.61$ ). We found significant evidence of small study effects for the maternal composite outcome (Peter's test $\mathrm{P}=0.04$ ), but not for the offspring composite outcome $(\mathrm{P}=0.85)$ (see web appendix 4). 


\begin{tabular}{|c|c|c|c|c|c|c|c|c|c|c|}
\hline \multirow[b]{2}{*}{ Outcomes } & \multicolumn{2}{|c|}{$\begin{array}{l}\text { No of studies } \\
\text { (No of women) }\end{array}$} & \multicolumn{2}{|c|}{$\begin{array}{l}\text { Intervention: } \\
\text { event/No event }\end{array}$} & \multicolumn{2}{|c|}{ Control: event/No event } & \multicolumn{2}{|l|}{ Odds ratio $(95 \% \mathrm{Cl})$} & \multicolumn{2}{|l|}{$I^{2}(\%)$} \\
\hline & IPD & $\begin{array}{l}\text { IPD and } \\
\text { non-IPD }\end{array}$ & IPD & $\begin{array}{l}\text { IPD and } \\
\text { non-IPD }\end{array}$ & IPD & $\begin{array}{l}\text { IPD and } \\
\text { non-IPD }\end{array}$ & IPD & $\begin{array}{l}\text { IPD and } \\
\text { non-IPD }\end{array}$ & IPD & $\begin{array}{l}\text { IPD and } \\
\text { non-IPD }\end{array}$ \\
\hline \multicolumn{11}{|l|}{ Maternal } \\
\hline \multicolumn{11}{|l|}{ Composite outcome: } \\
\hline Overall & $24(8851)$ & NA & $1896 / 2728$ & NA & $1837 / 2390$ & NA & 0.90 (0.79 to 1.03$)$ & NA & 26.7 & NA \\
\hline Diet & $3(397)$ & NA & $42 / 137$ & NA & $84 / 134$ & NA & $0.60(0.20$ to 1.75$)$ & NA & 0.0 & NA \\
\hline Physical activity & $9(2311)$ & NA & $346 / 850$ & NA & $367 / 748$ & NA & 0.81 (0.61 to 1.09$)$ & NA & 10.8 & NA \\
\hline Mixed approach & $13(6259)$ & NA & $1508 / 1742$ & NA & $1438 / 3009$ & NA & 0.97 (0.84 to 1.12 ) & NA & 34.9 & NA \\
\hline \multicolumn{11}{|l|}{ Gestational diabetes: } \\
\hline Overall & $27(9427)$ & $59(16885)$ & $584 / 4333$ & $974 / 7764$ & $571 / 3939$ & $1046 / 7101$ & 0.89 (0.72 to 1.10$)$ & $0.76(0.65$ to 0.89$)$ & 23.8 & 36.8 \\
\hline Diet & $4(490)$ & $8(1106)$ & $13 / 208$ & $57 / 476$ & $19 / 250$ & $75 / 498$ & 1.03 (0.30 to 3.61 ) & 0.79 (0.37 to 1.69$)$ & 0.0 & 0.0 \\
\hline Physical activity & $10(2700)$ & $27(6755)$ & $90 / 1300$ & $240 / 3153$ & $121 / 1189$ & $347 / 3015$ & 0.67 (0.46 to 0.99) & $0.66(0.53$ to 0.83$)$ & 0.0 & 0.0 \\
\hline Mixed approach & $14(6355)$ & $27(9342)$ & $481 / 2825$ & $677 / 4135$ & $441 / 2608$ & $672 / 3858$ & $1.02(0.79$ to 1.32$)$ & $0.88(0.72$ to 1.07$)$ & 35.2 & 10.8 \\
\hline \multicolumn{11}{|l|}{$\begin{array}{l}\text { Hypertensive } \\
\text { disorders of } \\
\text { pregnancy: }\end{array}$} \\
\hline Overall & $22(9618)$ & $45(14849)$ & $432 / 4586$ & $559 / 7130$ & $423 / 4177$ & $592 / 6568$ & $0.95(0.78$ to 1.16$)$ & $0.85(0.71$ to 1.00$)$ & 24.2 & 21.5 \\
\hline Diet & $3(397)$ & $5(729)$ & $18 / 161$ & $23 / 322$ & $39 / 179$ & $49 / 335$ & $0.59(0.07$ to 4.65$)$ & 0.57 (0.18 to 1.79$)$ & 35.8 & 38.0 \\
\hline Physical activity & $7(2565)$ & $20(5125)$ & $55 / 1242$ & $106 / 2513$ & $73 / 1195$ & $147 / 2359$ & $0.74(0.42$ to 1.33$)$ & $0.68(0.49$ to 0.93$)$ & 6.0 & 0.0 \\
\hline Mixed approach & $13(6797)$ & $21(9136)$ & $359 / 3183$ & $430 / 4295$ & $322 / 2933$ & $407 / 4004$ & 1.05 (0.86 to 1.28$)$ & $1.01(0.87 \text { to } 1.17)^{\star}$ & 19.4 & 16.3 \\
\hline \multicolumn{11}{|l|}{ Preterm birth: } \\
\hline Overall & $32(11676)$ & $49(14339)$ & $332 / 5713$ & $414 / 6971$ & $345 / 5286$ & $443 / 6511$ & 0.94 (0.78 to 1.13$)$ & $0.92(0.79$ to 1.08$)$ & 17.3 & 8.7 \\
\hline Diet & $4(1344)$ & $7(1696)$ & $9 / 647$ & $13 / 819$ & $35 / 653$ & $45 / 819$ & $0.28(0.08$ to 0.96$)$ & $0.32(0.14$ to 0.70$)$ & 0.0 & 0.0 \\
\hline Physical activity & $13(3249)$ & $23(5149)$ & $95 / 1541$ & $160 / 2431$ & $73 / 1540$ & $148 / 2410$ & $1.29(0.90$ to 1.85$)$ & $1.09(0.84$ to 1.41$)$ & 0.0 & 0.0 \\
\hline Mixed approach & $16(7219)$ & $20(7630)$ & $228 / 3525$ & $241 / 3721$ & $243 / 3223$ & $256 / 3412$ & 0.91 (0.73 to 1.12$)$ & $0.92(0.75$ to 1.12$)$ & 0.0 & 32.3 \\
\hline \multicolumn{11}{|l|}{ Caesarean section: } \\
\hline Overall & $32(11410)$ & 66 (18 041) & $1525 / 4385$ & $2373 / 6860$ & $1506 / 3994$ & $2440 / 6368$ & 0.91 (0.83 to 0.99$)$ & $0.89(0.83$ to 0.96$)$ & 0.0 & 16.2 \\
\hline Diet & $4(1340)$ & 7 (1732) & $117 / 535$ & $238 / 610$ & $149 / 539$ & $264 / 620$ & $0.78(0.50$ to 1.22$)$ & $0.88(0.65$ to 1.17$)$ & 0.0 & 0.0 \\
\hline Physical activity & $13(3046)$ & $32(6587)$ & $306 / 1230$ & $648 / 2646$ & $349 / 1161$ & $746 / 2547$ & $0.82(0.67$ to 1.01$)$ & $0.83(0.73$ to 0.95$)$ & 0.0 & 0.0 \\
\hline Mixed approach & $16(7160)$ & $28(9858)$ & $1102 / 2620$ & $1487 / 3604$ & $1059 / 2379$ & $1481 / 3286$ & 0.95 (0.84 to 1.08$)$ & $0.92(0.80$ to 1.06$)$ & 17.6 & 21.9 \\
\hline \multicolumn{11}{|l|}{ Offspring } \\
\hline \multicolumn{11}{|l|}{ Composite outcome: } \\
\hline Overall & $18(7981)$ & NA & $1007 / 3172$ & NA & $951 / 2851$ & NA & 0.94 (0.83 to 1.08$)$ & NA & 0.0 & NA \\
\hline Diet & $2(346)$ & NA & $34 / 132$ & NA & $48 / 132$ & NA & 0.71 (0.03 to 18.23$)$ & NA & 0.0 & NA \\
\hline Physical activity & $5(1274)$ & NA & $138 / 495$ & NA & $143 / 498$ & NA & 0.99 (0.67 to 1.46$)$ & NA & 0.0 & NA \\
\hline Mixed approach & $12(6494)$ & NA & $835 / 2545$ & NA & $797 / 2317$ & NA & $0.95(0.81$ to 1.11$)$ & NA & 4.7 & NA \\
\hline \multicolumn{11}{|l|}{ Stillbirtht: } \\
\hline Overall & $2(3719)$ & $4(4534)$ & $9 / 1858$ & $12 / 2261$ & $11 / 1841$ & $14 / 2247$ & 0.81 ( $(0.01$ to 256.69$)$ & $0.85(0.24$ to 3.02$)$ & 0.0 & 0.0 \\
\hline \multicolumn{11}{|l|}{$\begin{array}{l}\text { Small for gestational } \\
\text { age: }\end{array}$} \\
\hline Overall & $33(11666)$ & $44(12937)$ & $709 / 5324$ & $773 / 6018$ & $632 / 5001$ & $685 / 5461$ & $1.06(0.94$ to 1.20$)$ & 1.05 (0.94 to 1.18$)$ & 0.0 & 0.0 \\
\hline Diet & $4(1337)$ & $6(1628)$ & $41 / 610$ & $56 / 746$ & $47 / 639$ & $55 / 771$ & 0.92 (0.45 to 1.88$)$ & $1.05(0.62$ to 1.77$)$ & 0.0 & 0.0 \\
\hline Physical activity & $14(3272)$ & $21(3955)$ & $243 / 1402$ & $274 / 1740$ & $232 / 1395$ & $271 / 1670$ & $1.05(0.84$ to 1.34$)$ & $1.01(0.83$ to 1.24$)$ & 12.3 & 51.7 \\
\hline Mixed approach & $16(7193)$ & $20(7670)$ & $425 / 3312$ & $443 / 3532$ & $370 / 3086$ & $386 / 3309$ & $1.08(0.92$ to 1.28$)$ & $1.08(0.93$ to 1.27$)$ & 0.0 & 0.0 \\
\hline \multicolumn{11}{|l|}{$\begin{array}{l}\text { Large for gestational } \\
\text { age: }\end{array}$} \\
\hline Overall & $34(12047)$ & $45(13348)$ & $744 / 5492$ & $820 / 6185$ & $759 / 5052$ & $833 / 5510$ & $0.90(0.76$ to 1.07$)$ & $0.86(0.71$ to 1.04$)$ & 38.0 & 41.0 \\
\hline Diet & $4(1408)$ & $6(1699)$ & $155 / 529$ & $172 / 663$ & $176 / 548$ & $203 / 661$ & $0.91(0.60$ to 1.37$)$ & 0.82 (0.54 to 1.22$)$ & 0.0 & 0.0 \\
\hline Physical activity & $15(3330)$ & $21(3930)$ & $121 / 1557$ & $159 / 1842$ & $124 / 1528$ & $161 / 1768$ & $0.96(0.59$ to 1.54$)$ & $0.96(0.67$ to 1.37$)$ & 34.3 & 6.9 \\
\hline Mixed approach & $16(7450)$ & $21(8040)$ & $468 / 3406$ & $489 / 3680$ & $481 / 3095$ & $523 / 3348$ & 0.89 (0.67 to 1.17$)$ & $0.83(0.62$ to 1.10$)$ & 51.0 & 4.3 \\
\hline \multicolumn{11}{|c|}{$\begin{array}{l}\text { Admission to neonatal } \\
\text { intensive care unit: }\end{array}$} \\
\hline Overall & $16(8140)$ & $21(9498)$ & $302 / 3973$ & $406 / 4543$ & $279 / 3586$ & $400 / 4149$ & 1.01 (0.84 to 1.23$)$ & $0.97(0.82$ to 1.14$)$ & 0.0 & 0.0 \\
\hline Diet & $1(289)$ & $2(389)$ & $3 / 137$ & $11 / 179$ & $13 / 136$ & $29 / 170$ & NA & $0.33(<0.01$ to 47.97$)$ & NA & 0.0 \\
\hline Physical activity & $3(1166)$ & $4(1240)$ & $31 / 552$ & $34 / 586$ & $40 / 543$ & $43 / 577$ & 0.77 (0.21 to 2.81$)$ & $0.79(0.35$ to 1.78$)$ & 20.8 & 0.0 \\
\hline Mixed approach & $13(6818)$ & $15(7771)$ & $268 / 3284$ & $360 / 3626$ & $230 / 3036$ & $332 / 3453$ & $1.10(0.89$ to 1.35$)$ & $1.05(0.88$ to 1.25$)$ & 0.0 & 0.0 \\
\hline
\end{tabular}

\section{Discussion}

Our large, collaborative individual participant data (IPD) meta-analysis confirms that diet and physical activity based interventions in pregnancy reduce gestational weight gain. This beneficial effect was consistently observed irrespective of maternal body mass index (BMI), age, parity, ethnicity, or pre-existing medical condition; and remained when studies at high risk of bias were excluded. The findings are generalisable, with the $95 \%$ 


\begin{tabular}{|c|c|c|c|c|}
\hline Composite outcomes & $\begin{array}{l}\text { No of studies } \\
\text { (No of women) }\end{array}$ & $\begin{array}{l}\text { Odds ratio* } \\
(95 \% \mathrm{Cl})\end{array}$ & \multicolumn{2}{|l|}{ Treatment covariate interaction } \\
\hline \multicolumn{5}{|l|}{ Maternal } \\
\hline \multicolumn{5}{|l|}{ Baseline body mass index: } \\
\hline Normal & $12(2445)$ & $0.91(0.65$ to 1.28$)$ & \multirow[t]{2}{*}{$1.00 ; 0.98$ to 1.02 (0.98 to 1.02$) \dagger$} & \multirow[t]{2}{*}{0} \\
\hline Obese & $20(4181)$ & $0.92(0.80$ to 1.05$)$ & & \\
\hline \multicolumn{5}{|l|}{ Parity: } \\
\hline Nulliparous & $21(4613)$ & $0.87(0.71$ to 1.07$)$ & \multirow[t]{2}{*}{$1.03 ; 0.75$ to 1.39 (0.53 to 2.00$) \neq$} & \multirow[t]{2}{*}{34.0} \\
\hline Multiparous & $22(4186)$ & $0.92(0.78$ to 1.07$)$ & & \\
\hline \multicolumn{5}{|l|}{ Ethnicity: } \\
\hline$<20$ & $9(172)$ & $1.57(0.66$ to 3.71$)$ & $1.01 ; 0.99$ to 1.03 (0.99 to 1.03$)$ q & 0 \\
\hline \multicolumn{5}{|c|}{ Pre-existing medical condition**: } \\
\hline None & $15(3135)$ & 0.85 (0.66 to 1.09) & \multirow[t]{2}{*}{$1.44 ; 0.15$ to 13.74 (0.03 to 76.75$)+\dagger$} & \multirow[t]{2}{*}{24.9} \\
\hline$\geq 1$ & $5(89)$ & $1.65(0.36$ to 7.51$)$ & & \\
\hline \multicolumn{5}{|l|}{ Offspring } \\
\hline \multicolumn{5}{|l|}{ Baseline body mass index: } \\
\hline Normal & $7(1843)$ & $0.93(0.60$ to 1.43$)$ & \multirow[t]{3}{*}{$0.98 ; 0.95$ to 1.00 (0.94 to 1.02$) \dagger$} & \multirow[t]{3}{*}{18.5} \\
\hline Overweight & $12(2065)$ & $0.83(0.61$ to 1.13$)$ & & \\
\hline Obese & $13(4327)$ & $0.92(0.72$ to 1.19$)$ & & \\
\hline \multicolumn{5}{|l|}{ Parity: } \\
\hline$<20$ & $7(162)$ & $1.01(0.34$ to 2.98$)$ & & \\
\hline Pre-existing medical cond & & & & \\
\hline None & $12(3407)$ & 0.89 (0.74 to 1.08$)$ & $0.58 ; 0.03,9.81(<0.001$ to 2440.15$)+t$ & 0 \\
\hline$\geq 1$ & $3(63)$ & 0.54 (0.04 to 7.52$)$ & & \\
\hline 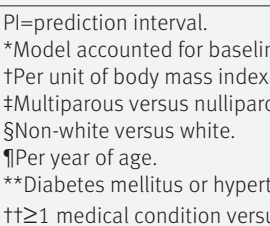 & nd clustering effect. & & & \\
\hline
\end{tabular}

prediction interval suggesting a beneficial effect on gestational weight gain when the intervention is applied in a new population or setting. There is no strong evidence that interventions reduce the risk of maternal and offspring composite outcomes, with no variation in effect observed across the subgroups.

For individual outcomes, interventions reduce caesarean section without a statistically significant reduction in other maternal and offspring complications. The effects of interventions for individual maternal and offspring complications are consistent irrespective of the BMI of the mother. Addition of study level data from non-IPD studies to the IPD meta-analysis increased the precision of estimates, without a change in the direction of effect, and showed additional benefit for gestational diabetes. Among individual interventions, those mainly based on physical activity lowered the odds of gestational diabetes.
Strengths and weaknesses of this study

To our knowledge this is the first IPD meta-analysis to assess the differential effects of diet and physical activity based interventions for important, clinically relevant outcomes, in subgroups of women who were identified a priori. Establishment of the International Weight Management in Pregnancy IPD Collaborative Group facilitated the collaboration of key researchers in this area and provided access to the largest IPD in this specialty. This allowed us to extract data that were not published, with larger sample sizes for outcomes such as preterm birth, small and large for gestational age fetuses, and admission to the neonatal intensive care unit for IPD than for study level metaanalysis. Furthermore, we were able to minimise the heterogeneity in the population by excluding individual women who did not fulfil the inclusion criteria. We compared the quality of studies that contributed to the IPD, which were generally of higher quality than those that did not contribute IPD. 
Access to IPD provided us with substantially increased power (compared with individual trials) to robustly estimate treatment covariate interactions and to avoid the ecological bias observed in aggregate metaregression of study level covariates. ${ }^{19} 21$ It also allowed us to adjust for baseline weight using analysis of covariance in each trial, ${ }^{134}$ which is the best approach to analysing continuous outcomes, ${ }^{135}$ although rarely used in individual trials. Our reporting of $95 \%$ prediction intervals for the overall and differential effects of interventions across subgroups allowed us to quantify the range of effects across populations of interest.

The subgroups were chosen in response to the call by the National Institute for Health and Care Excellence for assessment of the effectiveness of lifestyle interventions in pregnancy for specific groups of women considered to be at high risk of complications, such as teenagers, those from ethnic minorities, and women who enter pregnancy obese. ${ }^{15}$ We assessed treatment-covariate interactions for subgroups as both continuous and categorical variables. We chose 20 years as the cut-off for age, as it allowed us to assess the effect of intervention in young adults, where pregnancy may alter normal growth processes and increase the women's risk of becoming overweight or obese. ${ }^{136}$ Adolescent mothers also retain more weight post partum than mature control participants. ${ }^{136}$

Owing to the variation in reporting, we were only able to broadly classify the ethnicity of women as white or non-white. Our findings were limited by the smaller number of non-white compared with white mothers. We combined diet based, physical activity based, and mixed approach interventions to provide an overall estimate, and also reported their individual effects. ${ }^{13}$

${ }^{137}$ Since more than one clinical outcome is considered to be important to clinical care, we assessed the effects of interventions on maternal and offspring composite outcomes, the individual components of which were identified through a robust Delphi process. ${ }^{24}$ The varying definitions may have an impact on findings for gestational diabetes and pre-eclampsia, where the cut-offs and the criteria for diagnosis differed. Another limitation is that the majority of our population has a medium to high education status, a factor favouring compliance with interventions.

\section{IPD repository}

By establishing the International Weight Management in Pregnancy IPD live repository through the support of the individual research teams, we ensured that in addition to the standardisation, data were robustly safeguarded. The continuing growth of the repository is crucial for future research in this area ${ }^{138}$ and will accelerate update of the meta-analysis for the various relevant outcomes as new studies are published. We were successful in obtaining individual data from $80 \%$ of all participants within the IPD acquisition timeline. While every effort was made to include IPD from the latest studies identified in the updated search, we were limited by the time needed to prepare the IPD datasets, which involved data access, setting up of institutional contracts, cleaning and formatting of accessed data, resolution of queries with individual researchers, and standardisation and merging of the data. This restricted our ability to include studies published after the agreed data acquisition timeline in the IPD metaanalysis. In a high priority area such as obesity and weight gain in pregnancy, the number of published studies has increased rapidly, with at least 10 trials published each year since 2011, and 16 published in 2016. We sought to maximise the information needed to inform the findings by combining study level data from non-IPD studies with the IPD meta-analyses. The conclusions appeared to be robust for nearly all outcomes. Furthermore, the non-availability of IPD from these studies did not appear to contribute to the observed small study effects, since the asymmetry of the funnel plot was not altered when the nonIPD studies were added. Non-IPD studies were also generally at a higher risk of bias.

\section{Gestational weight gain}

Diet and physical activity based interventions reduce gestational weight gain. We have shown that this beneficial effect is observed in all women irrespective of maternal characteristics. The findings are consistent for any type of intervention, even when restricted to only high quality studies and to women adherent to the intervention, and when non-IPD are added to IPD. Mothers with excess weight gain in pregnancy are at increased risk of postpartum weight retention. ${ }^{139}$ This increase in interpregnancy BMI may contribute to risks of entering subsequent pregnancies as overweight or obese, with adverse outcomes in subsequent pregnancy. ${ }^{140}$ Furthermore, this may increase women's risk of cardiovascular morbidity and mortality in later life. ${ }^{141}$ Compared with published evidence, ${ }^{13}$ we identified a smaller reduction in gestational weight gain of $0.7 \mathrm{~kg}$ with interventions. The effect of such a reduction in gestational weight gain (compared with routine care) on postpartum weight retention and long term outcomes is not known.

\section{Maternal and offspring outcomes}

Despite the summary effect estimates favouring the interventions for maternal and offspring composite outcomes, these were not statistically significant. Interventions significantly reduced the odds of caesarean section. Previous systematic reviews showed a trend towards reduction in this risk overall, and for individual interventions (diet, physical activity, or mixed approach), ${ }^{13}$ but were limited by the small sample sizes and paucity of reporting, compared with the 11000 women included in our IPD meta-analysis. Of the individual interventions, physical activity in pregnancy showed a trend towards reduction in caesarean section in IPD meta-analysis, which became statistically significant with minimal heterogeneity when non-IPD were added. The physical activity component in most studies involved a structured exercise of moderate intensity (eg, aerobic classes or 
stationary cycling) with resistance training that varied in frequency (see web appendix 5). The relatively small numbers of women in the diet only intervention may have contributed to the imprecision in estimates.

Although the direction of effect appeared to favour the intervention for other maternal outcomes, they were not statistically significant. Addition of non-IPD to the IPD meta-analysis resulted in a statistically significant reduction in gestational diabetes. However, unlike our IPD analysis, we were not able to implement the strict inclusion and exclusion criteria, standardise the analysis strategy (eg, adjust for baseline), or ascertain occurrence of outcome in the combined analysis with study level data. Physical activity based interventions statistically significantly reduced the odds of gestational diabetes in IPD meta-analysis, and also when combined with non-IPD. This benefit could be mediated through mechanisms that resulted in improved glycaemic variables and outcomes in type 4 and type 2 diabetes, through increased insulin sensitivity and reduced oxidative stress. Exercise in pregnancy may also have a potential role in preventing hypertensive disorders of pregnancy. The effects of diet and physical activity on maternal and offspring outcomes did not vary according to the BMI of the woman, highlighting the potential benefits for all and not selected groups of mothers.

Interventions based on diet showed a reduction in preterm birth, although the analysis included relatively small numbers of women. We did not identify any benefits with interventions in preventing any adverse offspring outcome, despite a sample size that was twofold to threefold more than published data for some outcomes, consistent with previous findings. ${ }^{14}$ The lack of adverse effects such as small for gestational age and preterm birth with diet and physical activity in pregnancy should reassure mothers who have traditionally been advised not to undertake structured exercise or manage their diet in pregnancy.

\section{Implications for clinical practice}

Currently in the UK, only obese women are offered access to a dietician and specific antenatal classes for advice on diet and lifestyle, to minimise gestational weight gain. Based on our work, it is likely that women of all BMI groups could benefit from specific advice on diet and physical activity for weight gain, and some maternal outcomes. Healthcare professionals should avoid variations in care and lifestyle advice provided to mothers based on ethnicity, age, and underlying medical conditions, as no differential effects were found.

Discussions about diet and physical activity in pregnancy, which are delivered as part of antenatal care, should incorporate specific estimates of benefit for caesarean section and gestational weight gain, and the likelihood of preventing gestational diabetes. Mothers should be reassured about the safety of the interventions, particularly on physical activity and structured exercise in pregnancy, by highlighting the benefits and lack of harm. This may improve engagement and compliance with the intervention. Importantly, such interventions in pregnancy could be considered in global efforts to reduce caesarean section in relevant populations.

\section{Implications for further research}

Whether the observed benefit in gestational weight gain with diet and physical activity translates to long term benefits to the mother and child needs to be assessed. Evaluation of any differential effects according to the individual components of the intervention, such as duration, frequency, provider, and setting, on individual outcomes is required to provide detailed recommendations. The effects of these interventions on mothers in low and middle income countries, particularly in those countries with high rates of caesarean section and gestational diabetes, need to be ascertained from large randomised trials. There is a need to develop a harmonised core outcome set for future reporting of clinical trials in this area, to maximise the meaningful interpretation of published data. This is particularly relevant for rare but important outcomes such as shoulder dystocia, birth trauma, and venous thromboembolic events.

\section{Conclusion}

Diet and physical activity based interventions in pregnancy limit gestational weight gain, with no evidence that this effect differs across subgroups defined by maternal characteristics. The odds for caesarean section are also reduced.

We thank all researchers, research nurses, and staff of the participating centres in the trials contributing to this IPD metaanalysis. We thank Erica Harris for her input as a patient group representative. Members of the International Weight Management in Pregnancy (i-WIP) Collaborative Group: Ewelina Rogozińska, doctoral researcher, Nadine Marlin, statistician, Ana Pilar Betrán, medical officer, Arne Astrup, professor, Annick Bogaerts, assistant professor, Jose G Cecatti, professor, Roland Devlieger, associate professor, Jodie M Dodd, professor, Nermeen El Beltagy, associate professor, Fabio Facchinetti, professor, chairman of the unit, Nina RW Geiker, senior researcher, Kym J Guelfi, senior lecturer, Lene AH Haakstad, associate professor, Cheryce L Harrison, research fellow, Hans Hauner, professor, Dorte M Jensen, consultant, assistant professor, Tarja I Kinnunen, lecturer, Janette Khoury, researcher, Riitta Luoto, research director, associate professor, Fionnuala McAuliffe, professor, Narges Motahari, reseaarcher, Siv Mørkved, professor, Julie Owens, professor María Perales, research fellow, Elisabetta Petrella, medical doctor, Suzanne Phelan, professor, Lucilla Poston, professor, Kathrin Rauh, research fellow, Kristina M Renault, obstetrician, Linda R Sagedal, physician, Kjell Å Salvesen, professor, Garry X Shen, professor, Alexis Shub, obstetrician, senior lecturer, Tânia Scudeller, professor, Fernanda G Surita, associate professor, Signe N Stafne, postdoctoral research fellow, Helena Teede, director, professor, Serena Tonstad, head physician, Mireille NM van Poppel, professor, Christina A Vinter, obstetrician, Ingvild Vistad, consultant, SeonAe Yeo, professor, Julie Dodds, senior research manager, Sally Kerry, reader, Louise Jackson, research fellow, Pelham Barton, reader, Emma Molyneaux, postdoctoral research fellow, Alba A Martin, research fellow, Girish Rayanagoudar, research fellow, Anneloes E Ruifrok, resident, Tracy Roberts, professor, Christianne JM de Groot, professor, Arri Coomarasamy, professor, Ben WJ Mol, professor, Javier Zamora, senior lecturer, Khalid S Khan, professor, Richard D Riley, professor, Shakila Thangaratinam, professor (see web appendix 6).

Contributors: ST, RDR, CdG, AER, and SK developed the protocol. ID oversaw the project and drafted the manuscript. ST, ER, and NM conducted the review, drafted the manuscript, and led the project. KSK and BWJM provided input into the development of the protocol and drafting of the initial manuscript. ER, EM, and AAM undertook the literature searches and study selection. AER, ER, ST, EM, and GR acquired IPD. MvP, LP, CAV, FMCA, JMD, JO, RB, MP, JGC, FGS, SY, AB, RD, HT, CLH, LH, GXS, AS, NEB, NMo, JK, STo, RL, TIK, KJG, FF, EP, SP, TS, KR, 
HH, KMR, LRS, IV, SNS, SM, KAS, DMJ, MvP, AA, and NRWG contributed data to the project and provided input at all stages of the project. ER, $\mathrm{GR}$, and NM mapped the variables in the available datasets. ER and NM cleaned and quality checked data. NM harmonised the data. NM, SK, and RDR conducted the data analysis. TR, LJ, and PB provided input into the protocol. APB provided input into the conduct of study. IZ provided methodological support. KSK, AC, and BWIM were involved in project development and provided input at all stages. All authors critically appraised the final draft of the report. ST is the guarantor.

Funding: This study was funded by the National Institute for Health Research (NIHR) Health Technology Assessment) programme (No 12/01/50). The NIHR had no influence on the study design, its conduct, interpretation of study findings, writing of the manuscript; and in the decision to submit the article for publication.

Competing interests: All authors have completed the ICMJE uniform disclosure form at www.icmje.org/coi disclosure.pdf and declare: no support from any organisation for the submitted work. HH reports grants from the German Ministry of Education and Research, the Bavarian Ministry of Agriculture and Nutrition, the Bavarian Ministry of Health, the Helmholtz Center Munich, the Else Kröner-Fresenius Foundation, AOK Bavaria, Amway, and the German Research Foundation outside the submitted work. BWJM reports funding from ObsEva during the conduct of the study.

Ethical approval: Not required.

Data sharing: The full dataset or its subset and technical appendix are available from the data custodian (Queen Mary University of London) at smd-iwipdata@gmul.ac.uk. Access to the datase is regulated by terms and conditions available on request. The presented data are anonymised and risk of identification of individual participants is low.

Transparency: The lead author (IER) affirms that the manuscript is an honest, accurate, and transparent account of the study being reported; that no important aspects of the study have been omitted; and that any discrepancies from the study as planned have been explained.

This is an Open Access article distributed in accordance with the Creative Commons Attribution Non Commercial (CC BY-NC 4.0) license, which permits others to distribute, remix, adapt, build upon this work non-commercially, and license their derivative works on different terms, provided the original work is properly cited and the use is noncommercial. See: http://creativecommons.org/licenses/by-nc/4.0/.

1 Branum AMKS, Kirmeyer SE, Gregory EC. Prepregnancy Body Mass Index by Maternal Characteristics and State: Data From the Birth Certificate, 2014. Natl Vital Stat Rep 2016:65:1-11.

2 England PH. UK prevalence of maternal obesity. 2016. www.noo. org.uk/NOO_about_obesity/maternal_obesity_2015/prevalence (Accessed 23 Aug 2016).

3 Flegal KM, Kruszon-Moran D, Carroll MD, Fryar CD, Ogden CL. Trends in Obesity Among Adults in the United States, 2005 to 2014. JAMA 2016;315:2284-91. doi:10.1001/jama.2016.6458

4 Johansson S, Villamor E, Altman M, Bonamy AK, Granath F, Cnattingius S. Maternal overweight and obesity in early pregnancy and risk of infant mortality: a population based cohort study in Sweden. BM/ 2014:349:g6572. doi:10.1136/bmj.g6572

5 Leddy MA, Power ML, Schulkin J. The impact of maternal obesity on maternal and fetal health. Rev Obstet Gynecol 2008;1:170-8.

6 Viteri OA, Salazar XC, Refuerzo IS. Maternal and Infant Implications of Excessive Gestational Weight Gain among Obese Pregnant Women. J Endocrinol Diabetes Obes 2015;3:8.

7 Morgan KL, Rahman MA, Macey S. Obesity in pregnancy: a retrospective prevalence-based study on health service utilisation and costs on the NHS. BMJ Open 2014;4:e003983. doi:10.1136/ bmjopen-2013-003983

8 Rtveladze K, Marsh T. Webber L. Health and economic burden of obesity in Brazil. PLoS One 2013;8:e68785. doi:10.1371/journal. pone. 0068785

9 CMACE. Maternal obesity in the UK: findings from a national project.CMACE, 2010

10 Khalid K, Thangaratinam S, Coomarasamy A, et al. HTA 09/27/06 Interventions to reduce or prevent obesity in pregnant women: a systematic review. UK/Poland: National Institute for HEalth Research; 2010.

11 Poston L. Improving pregnancy outcome in obese women. National Institute for Health Research, 2008

12 Thangaratinam S, Riley R, Kerry S. HTA - 12/01/50: Effects of weight management interventions on maternal and fetal outcomes in pregnancy: Individual patient data (IPD) meta analysis of randomised trials and model based economic evaluation. National Institute for Health Research, 2013.
13 Thangaratinam S, Rogozinska E, Jolly K. Effects of interventions in pregnancy on maternal weight and obstetric outcomes: meta-analysis of randomised evidence. BM/ 2012;344:e2088. doi:10.1136/bmj. e2088

14 Muktabhant B LT, Lumbiganon P, Laopaiboon M. Diet or exercise, or both, for preventing excessive weight gain in pregnancy. Cochrane Database of Systematic Reviews 2015;(6):CD007145.

15 NICE. Weight management before, during and after pregnancy. National Institute for Health and Clinical Excellence, 2010.

16 Dodd JM, Turnbull D, McPhee AJLIMIT Randomised Trial Group. Antenatal lifestyle advice for women who are overweight or obese: LIMIT randomised trial. BMJ 2014;348:g1285. doi:10.1136/bmj. g1285.

17 Poston L, Bell R, Croker HUPBEAT Trial Consortium. Effect of a behavioural intervention in obese pregnant women (the UPBEAT study): a multicentre, randomised controlled trial. Lancet Diabetes Endocrinol 2015;3:767-77. doi:10.1016/S2213-8587(15)00227-2

18 Thangaratinam S, Rogozińska E, Jolly K. Interventions to reduce or prevent obesity in pregnant women: a systematic review. Health Technol Assess 2012;16:iii-iv, 1-191. doi:10.3310/hta16310

19 Berlin JA, Santanna J, Schmid CH, Szczech LA, Feldman HIAntiLymphocyte Antibody Induction Therapy Study Group. Individual patient- versus group-level data meta-regressions for the investigation of treatment effect modifiers: ecological bias rears its ugly head. Stat Med 2002;21:371-87. doi:10.1002/sim.1023

20 Lambert PC, Sutton AJ, Abrams KR, Jones DR. A comparison of summary patient-level covariates in meta-regression with individual patient data meta-analysis. J Clin Epidemiol 2002;55:86-94. doi:10.1016/S0895-4356(01)00414-0

21 Riley RD, Lambert PC, Abo-Zaid G. Meta-analysis of individual participant data: rationale, conduct, and reporting. $B M$ 2010:340:c221. doi:10.1136/bmi.c221

22 Ruifrok AE, Rogozinska E, van Poppel MNi-WIP (International Weight Management in Pregnancy) Collaborative Group. Study protocol: differential effects of diet and physical activity based interventions in pregnancy on maternal and fetal outcomes--individual patient data (IPD) meta-analysis and health economic evaluation. Syst Rev 2014;3:131. doi:10.1186/2046-4053-3-131

23 Stewart LA, Clarke M, Rovers MPRISMA-IPD Development Group. Preferred Reporting Items for Systematic Review and Meta-Analyses of individual participant data: the PRISMA-IPD Statement. JAMA 2015;313:1657-65. doi:10.1001/jama.2015.3656

24 Rogozinska E, D’Amico MI, Khan KSInternational Weight Management in Pregnancy (iWIP) Collaborative Group. Development of composite outcomes for individual patient data (IPD) meta-analysis on the effects of diet and lifestyle in pregnancy: a Delphi survey. BJOG 2016;123:190-8. doi:10.1111/1471-0528.13764

25 Gardosi J FA. GROW calculator v6.7.7. 2016

26 i-WIP Group, 2013 kamolo.org.ar/iwipipd/index.asp (accessed 5/ Sep/2016)

27 Higgins JP, Altman DG. Assessing risk of bias in included studies. In: Higgins JPTGS, ed. Cochrane Handbook for Systematic Reviews of Interventions.John Wiley \& Sons, 2008doi:10.1002/9780470712184.ch8.

28 Cornell JE, Mulrow CD, Localio R. Random-effects meta-analysis of inconsistent effects: a time for change. Ann Intern Med 2014;160:267-70. doi:10.7326/M13-2886

29 Hartung J, Knapp G. A refined method for the meta-analysis of controlled clinical trials with binary outcome. Stat Med 2001:20:3875-89. doi:10.1002/sim.1009

30 Higgins JP, Thompson SG, Deeks JJ, Altman DG. Measuring inconsistency in meta-analyses. BMJ 2003;327:557-60. doi:10.1136/bmi.327.7414.557

31 Riley RD, Higgins JP, Deeks JJ. Interpretation of random effects metaanalyses. BMJ 2011;342:d549. doi:10.1136/bmj.d549

32 Sterne IA, Sutton AJ, Ioannidis IP. Recommendations for examining and interpreting funnel plot asymmetry in meta-analyses of randomised controlled trials. BMJ 2011;343:d4002. doi:10.1136/ bmi.d4002

33 Ahmed I, Sutton AJ, Riley RD. Assessment of publication bias, selection bias, and unavailable data in meta-analyses using individual participant data: a database survey. BMJ 2012;344:d7762.

34 Althuizen E, van der Wijden CL, van Mechelen W, Seidell JC, van Poppel MN. The effect of a counselling intervention on weight changes during and after pregnancy: a randomised trial. BJOG 2013:120:92-9 doi:10.1111/1471-0528.12014

35 Baciuk EP, Pereira RI, Cecatti JG, Braga AF, Cavalcante SR. Water aerobics in pregnancy: Cardiovascular response, labor and neonatal outcomes. Reprod Health 2008:5:10. doi:10.1186/1742-4755-510

36 Barakat R, Stirling JR, Lucia A. Does exercise training during pregnancy affect gestational age? A randomised controlled trial. $\mathrm{Br}$ ) Sports Med 2008;42:674-8. doi:10.1136/bjsm.2008.047837 
37 Barakat R, Pelaez M, Montejo R, Luaces M, Zakynthinaki M. Exercise during pregnancy improves maternal health perception: a randomized controlled trial. Am J Obstet Gynecol 2011;204:402. e1-7. doi:10.1016/j.ajog.2011.01.043

38 Barakat R, Pelaez M, Lopez C, Montejo R, Coteron J. Exercise during pregnancy reduces the rate of cesarean and instrumental deliveries: results of a randomized controlled trial. / Matern Fetal Neonatal Med 2012;25:2372-6. doi:10.3109/14767058.2012.696165

39 Bogaerts AF, Devlieger R, Nuyts E, Witters I, Gyselaers W, Van den Bergh BR. Effects of lifestyle intervention in obese pregnant women on gestational weight gain and mental health: a randomized controlled trial. Int / Obes (Lond) 2013;37:814-21. doi:10.1038/ijo.2012.162

40 El Beltagy N, Saad El Deen S, Mohamed R. Does physical activity and diet control reduce the risk of developing gestational diabetes mellitus in Egypt? A randomized controlled trial. J Perinat Med 2013:41. doi:10.1515/ipm-2013-2002

41 Guelinckx I, Devlieger R, Mullie P, Vansant G. Effect of lifestyle intervention on dietary habits, physical activity, and gestational weight gain in obese pregnant women: a randomized controlled trial. Am J Clin Nutr 2010;91:373-80. doi:10.3945/ajcn.2009.28166

42 Haakstad LA, B $\varnothing$ K. Effect of regular exercise on prevention of excessive weight gain in pregnancy: a randomised controlled trial. Eur J Contracept Reprod Health Care 2011;16:116-25. doi:10.3109/ 13625187.2011.560307

43 Harrison CL, Lombard CB, Strauss BJ, Teede HJ. Optimizing healthy gestational weight gain in women at high risk of gestational diabetes: a randomized controlled trial. Obesity (Silver Spring) 2013;21:9049. doi:10.1002/oby.20163

44 Hui A, Back L, Ludwig S. Lifestyle intervention on diet and exercise reduced excessive gestational weight gain in pregnant women unde a randomised controlled trial. BJOG 2012;119:70-7. doi:10.1111/ j.1471-0528.2011.03184.X

45 Jeffries K, Shub A, Walker SP, Hiscock R, Permezel M. Reducing excessive weight gain in pregnancy: a randomised controlled trial. Med J Aust 2009;191:429-33.

46 Khaledan A, Motahari Tabari N, Ahmad Shirvani M. Effect of an Aerobic Exercise Program on Fetal Growth in Pregnant Women. HAYAT 2010;16:55-64. http://hayat.tums.ac.ir/browse.php?a id $=98 \&$ sid $=1 \&$ slc lang=en

47 Khoury J, Henriksen T, Christophersen B, Tonstad S. Effect of a cholesterol-lowering diet on maternal, cord, and neonatal lipids, and pregnancy outcome: a randomized clinical trial. Am J Obstet Gynecol 2005:193:1292-301. doi:10.1016/j.ajog.2005.05.016

48 Luoto R, Kinnunen TI, Aittasalo M. Primary prevention of gestational diabetes mellitus and large-for-gestational-age newborns by lifestyle counseling: a cluster-randomized controlled trial. PLoS Med 2011;8:e1001036. doi:10.1371/journal.pmed.1001036

49 Nascimento SL, Surita FG, Parpinelli MÂA, Siani S, Pinto e Silva JL. The effect of an antenatal physical exercise programme on maternal/ perinatal outcomes and quality of life in overweight and obese pregnant women: a randomised clinical trial. BJOG 2011;118:145563. doi:10.1111/j.1471-0528.2011.03084.x

50 Ong MJ, Guelfi KJ, Hunter T, Wallman KE, Fournier PA, Newnham JP. Supervised home-based exercise may attenuate the decline of glucose tolerance in obese pregnant women. Diabetes Metab 2009;35:418-21. doi:10.1016/i.diabet.2009.04.008

51 Oostdam N, van Poppel MNM, Wouters MGAJ. No effect of the FitFor2 exercise programme on blood glucose, insulin sensitivity, and birthweight in pregnant women who were overweight and at risk for gestational diabetes: results of a randomised controlled trial. BJOG 2012;119:1098-107. doi:10.1111/j.1471-0528.2012.03366.x

52 Perales M, Refoyo I, Coteron J, Bacchi M, Barakat R. Exercise during pregnancy attenuates prenatal depression: a randomized controlled trial. Eval Health Prof 2015;38:59-72. doi:10.1177/0163278714533566

53 Petrella E, Malavolti M, Bertarini V. Gestational weight gain in overweight and obese women enrolled in a healthy lifestyle and eating habits program. J Matern Fetal Neonatal Med 2014;27:1348 52. doi:10.3109/14767058.2013.858318

54 Phelan S, Phipps MG, Abrams B, Darroch F, Schaffner A, Wing RR. Randomized trial of a behavioral intervention to prevent excessive gestational weight gain: the Fit for Delivery Study. Am J Clin Nutr 2011:93:772-9. doi:10.3945/ajcn.110.005306

55 Prevedel TCl, DeConti M, Adami HO. Maternal and perinatal effects of hydrotherapy in pregnancy. Rev Bras Ginecol Obstet 2003;25:53-9.

56 Rauh K, Gabriel E, Kerschbaum E. Safety and efficacy of a lifestyle intervention for pregnant women to prevent excessive maternal weight gain: a cluster-randomized controlled trial. BMC Pregnancy Childbirth 2013;13:151. doi:10.1186/1471-2393-13-151

57 Renault KM, Nørgaard K, Nilas L. The Treatment of Obese Pregnant Women (TOP) study: a randomized controlled trial of the effect of physical activity intervention assessed by pedometer with or without dietary intervention in obese pregnant women. Am / Obstet Gynecol 2014;210:134.e1-9. doi:10.1016/j.ajog.2013.09.029
58 Ruiz JR, Perales M, Pelaez M, Lopez C, Lucia A, Barakat R. Supervised exercise-based intervention to prevent excessive gestational weight gain: a randomized controlled trial. Mayo Clin Proc 2013;88:138897. doi:10.1016/j.mayocp.2013.07.020

59 Stafne SN, Salvesen KA, Romundstad PR, Torjusen IH, Mørkved S. Does regular exercise including pelvic floor muscle training prevent urinary and anal incontinence during pregnancy? A randomised controlled trial. BJOG 2012;119:1270-80. doi:10.1111/j.14710528.2012.03426.x

60 Sagedal LR, Øverby NC, Bere E. Lifestyle intervention to limit gestational weight gain: the Norwegian Fit for Delivery randomised controlled trial. BJOG 2017;124:97-109. doi:10.1111/14710528.13862

61 Walsh JM, McGowan CA, Mahony R, Foley ME, McAuliffe FM. Low glycaemic index diet in pregnancy to prevent macrosomia ROLO study): randomised control trial. BMJ 2012;345:e5605. doi:10.1136/bmj.e5605

62 Wolff S, Legarth J, Vangsgaard K, Toubro S, Astrup A. A randomized trial of the effects of dietary counseling on gestational weight gain and glucose metabolism in obese pregnant women. Int J Obes (Lond) 2008;32:495-501. doi:10.1038/sj.ijo.0803710

63 Vinter CA, Jensen DM, Ovesen P, Beck-Nielsen H, Jørgensen IS. The LiP (Lifestyle in Pregnancy) study: a randomized controlled trial of lifestyle intervention in 360 obese pregnant women. Diabetes Care 2011;34:2502-7. doi:10.2337/dc11-1150

64 Vítolo MR, Bueno MS, Gama CM. [Impact of a dietary counseling program on the gain weight speed of pregnant women attended in a primary care service]. Rev Bras Ginecol Obstet 2011;33:13-9.

65 Yeo S, Neelon V, Weaver M, et al. Regular exercise from 12-22 weeks of pregnancy in women at risk for preeclampsia: A feasibility study. (unpublished)

66 Yeo S, Steele NM, Chang MC, Leclaire SM, Ronis DL, Hayashi R. Effect of exercise on blood pressure in pregnant women with a high risk of gestational hypertensive disorders. J Reprod Med 2000;45:293-8

67 Perales M, Calabria I, Lopez C, Franco E, Coteron J, Barakat R. Regula Exercise Throughout Pregnancy Is Associated With a Shorter Firs Stage of Labor. Am J Health Promot 2016;30:149-54. doi:10.4278/ ajhp.140221-QUAN-79

68 Badrawi H, Hassanein MK, Badraoui MHH. Pregnancy outcome in obese pregnant mothers. J Perinat Med 1992;20((Suppl 1):203.

69 Barakat R, Cordero Y, Coteron J, Luaces M, Montejo R. Exercise during pregnancy improves maternal glucose screen at 24-28 weeks: randomised controlled trial. Br J Sports Med 2012;46:656-61. doi:10.1136/bjsports-2011-090009

70 Barakat R, Pelaez M, Lopez C, Lucia A, Ruiz IR. Exercise during pregnancy and gestational diabetes-related adverse effects: a randomised controlled trial. Br J Sports Med 2013;47:630-6. doi:10.1136/bjsports-2012-091788

71 Bechtel-Blackwell DA. Computer-assisted self-interview and nutrition education in pregnant teens. Clin Nurs Res 2002;11:450-62. doi:10.1177/105477302237456

72 Briley C, Flanagan NL, Lewis N. In-home prenatal nutrition intervention increased dietary iron intakes and reduced low birthweight in low-income African-American women. J Am Diet Assoc 2002;102:984-7. doi:10.1016/S0002-8223(02)90225-7

73 Clapp JF 3rd, Kim H, Burciu B, Lopez B. Beginning regular exercise in early pregnancy: effect on fetoplacental growth. Am J Obstet Gynecol 2000;183:1484-8. doi:10.1067/mob.2000.107096

74 Deveer R, Deveer M, Akbaba E. The effect of diet on pregnancy outcomes among pregnant with abnormal glucose challenge test. Eur Rev Med Pharmacol Sci 2013;17:1258-61.

75 Garshasbi A, Faghih Zadeh S. The effect of exercise on the intensity of low back pain in pregnant women. Int J Gynaecol Obstet 2005;88:271-5. doi:10.1016/j.ijgo.2004.12.001

76 Gomez-Tabarez G, Delgado JG, Agudelo AA. Diet effects on the perinatal result of obese pregnant patient[Spanish]. Rev Colomb Obstet Ginecol 1994;45:313-6.

77 Hopkins SA, Baldi IC, Cutfield WS, McCowan L, Hofman PL. Exercise training in pregnancy reduces offspring size without changes in maternal insulin sensitivity. J Clin Endocrinol Metab 2010;95:20808. doi:10.1210/jc.2009-2255

78 Huang TT, Yeh CY, Tsai YC. A diet and physical activity intervention for preventing weight retention among Taiwanese childbearing women: a randomised controlled trial. Midwifery 2011;27:257-64. doi:10.1016/j.midw.2009.06.009

79 Lee G, Challenger S. Exercise in pregnancy. Mod Midwife 1996;6:2833

80 Jackson RA, Stotland NE, Caughey AB, Gerbert B. Improving diet and exercise in pregnancy with Video Doctor counseling: a randomized trial. Patient Educ Couns 2011;83:203-9. doi:10.1016/j. pec.2010.05.019

81 Korpi-Hyövälti E, Schwab U, Laaksonen DE, Linjama H, Heinonen S, Niskanen L. Effect of intensive counselling on the quality of dietary 
fats in pregnant women at high risk of gestational diabetes mellitus. Br J Nutr 2012;108:910-7. doi:10.1017/S0007114511006118

82 Marquez-Sterling S, Perry AC, Kaplan TA, Halberstein RA, Signorile JF. Physical and psychological changes with vigorous exercise in sedentary primigravidae. Med Sci Sports Exerc 2000;32:58-62. doi:10.1097/00005768-200001000-00010

83 Polley BA, Wing RR, Sims C). Randomized controlled trial to prevent excessive weight gain in pregnant women. Int J Obes Relat Metab Disord 2002:26:1494-502. doi:10.1038/sj.ijo.0802130

84 Quinlivan JA, Lam LT, Fisher J. A randomised trial of a four-step multidisciplinary approach to the antenatal care of obese pregnant women. Aust N Z J Obstet Gynaecol 2011;51:141-6. doi:10.1111/ j.1479-828X.2010.01268.X

85 Santos IA, Stein R, Fuchs SC. Aerobic exercise and submaximal functional capacity in overweight pregnant women: a randomized trial. Obstet Gynecol 2005;106:243-9. doi:10.1097/01. AOG.0000171113.36624.86

86 Sedaghati P, Ziaee V, Ardjmand A. The effect of an ergometric training program on pregnants weight gain and low back pain. Gazzetta Med Ital Arch Sci Med 2007;166:209-13

87 Thornton YS, Smarkola C, Kopacz SM, Ishoof SB. Perinatal outcomes in nutritionally monitored obese pregnant women: a randomized clinical trial. / Natl Med Assoc 2009;101:569-77. doi:10.1016/ S0027-9684(15)30942-1

88 Vesco K, Leo M, Gillman M. Impact of a weight management intervention on pregnancy outcomes among obese women: The Healthy Moms Trial. Am J Obstet Gynecol 2013;208:S352doi:10.1016/j.ajog.2012.12.009.

89 Barakat R, Pelaez M, Cordero Y. Exercise during pregnancy protects against hypertension and macrosomia: randomized clinical trial. Am J Obstet Gynecol 2016;214:649.e1-8. doi:10.1016/j. ajog.2015.11.039.

90 Barakat R, Perales M, Bacchi M, Coteron J, Refoyo I. A program of exercise throughout pregnancy. Is it safe to mother and newborn?Am J Health Promot 2014;29:2-8. doi:10.4278/ajhp.130131-QUAN-56

91 Bisson M, Alméras N, Dufresne SS. A 12-Week Exercise Program for Pregnant Women with Obesity to Improve Physical Activity Levels: An Open Randomised Preliminary Study. PLoS One 2015;10:e0137742. doi:10.1371/journal.pone.0137742

92 Brownfoot FC, Davey MA, Kornman L. Routine weighing to reduce excessive antenatal weight gain: a randomised controlled trial. BJOG 2016;123:254-61. doi:10.1111/1471-0528.13735

93 Cordero Y, Mottola MF, Vargas J, Blanco M, Barakat R. Exercise Is Associated with a Reduction in Gestational Diabetes Mellitus. Med Sci Sports Exerc 2015;47:1328-33. doi:10.1249/ MSS.0000000000000547

94 Daley AJ, Jolly K, Jebb SA. Feasibility and acceptability of regular weighing, setting weight gain limits and providing feedback by community midwives to prevent excess weight gain during pregnancy: randomised controlled trial and qualitative study. $B M C$ Obes 2015;2:35. doi:10.1186/s40608-015-0061-5

95 Das SK, Roberts S, Saltzman E. Effect of a Behavioral Intervention with Cereal Fiber or Resistant Starch on Gestational Weight Gain: A Randomized Clinical Trial. FASEB J 2015;29(Suppl 1):117.2.

96 de Oliveria Melo AS, Silva JL, Tavares JS, Barros VO, Leite DF, Amorim MM. Effect of a physical exercise program during pregnancy on uteroplacental and fetal blood flow and fetal growth: a randomized controlled trial. Obstet Gynecol 2012;120:302-10. doi:10.1097/ AOG.0b013e31825de592

97 Dekker Nitert M, Barrett HL, Denny KJ, McIntyre HD, Callaway LKBAMBINO group. Exercise in pregnancy does not alter gestational weight gain, MCP-1 or leptin in obese women. Aust N Z J Obstet Gynaecol 2015;55:27-33. doi:10.1111/ajo.12300

98 Di Carlo C, lannotti G, Sparice S. The role of a personalized dietary intervention in managing gestational weight gain: a prospective, controlled study in a low-risk antenatal population. Arch Gynecol Obstet 2014;289:765-70. doi:10.1007/s00404-013-3054-y

99 Gesell SB, Katula JA, Strickland C, Vitolins MZ. Feasibility and Initial Efficacy Evaluation of a Community-Based Cognitive-Behavioral Lifestyle Intervention to Prevent Excessive Weight Gain During Pregnancy in Latina Women. Matern Child Health J 2015;19:184252. doi:10.1007/s10995-015-1698-x

100 Hawkins M, Hosker M, Marcus BH. A pregnancy lifestyle intervention to prevent gestational diabetes risk factors in overweight Hispanic women: a feasibility randomized controlled trial. Diabet Med 2015:32:108-15. doi:10.1111/dme.12601

101 Herring SJ, Cruice JF, Bennett GG, Rose MZ, Davey A, Foster GD. Preventing excessive gestational weight gain among African American women: A randomized clinical trial. Obesity (Silver Spring) 2016;24:30-6. doi:10.1002/oby.21240

102 Hui AL, Back L, Ludwig S. Effects of lifestyle intervention on dietary intake, physical activity level, and gestational weight gain in pregnant women with different pre-pregnancy Body Mass Index in a randomized control trial. BMC Pregnancy Childbirth 2014;14:331. doi:10.1186/1471-2393-14-331
103 Jing W, Huang Y, Liu X, Luo B, Yang Y, Liao S. The effect of a personalized intervention on weight gain and physical activity among pregnant women in China. Int J Gynaecol Obstet 2015;129:138-41. doi:10.1016/j.ijgo.2014.11.014

104 Koivusalo SB, Rönö K, Klemetti MM. Gestational Diabetes Mellitus Can Be Prevented by Lifestyle Intervention: The Finnish Gestationa Diabetes Prevention Study (RADIEL): A Randomized Controlled Trial. Diabetes Care 2016;39:24-30. doi:10.2337/dc15-0511

105 Kong KL, Campbell CG, Foster RC, Peterson AD, Lanningham-Foste L. A pilot walking program promotes moderate-intensity physical activity during pregnancy. Med Sci Sports Exerc 2014;46:462-71. doi:10.1249/MSS.0000000000000141

106 Li Q, Cui H, Zheng D, Li N, Chang L, Liu C. [Effects of walking exercise during late trimester on pregnancy outcome of low-risk primipara]. Zhonghua Yi Xue Za Zhi 2014;94:1722-5.

107 Mujsindi W, Habash D, Childs G. Impact of nutrition education on gestational weight gain in obese pregnant women. Am J Obstet Gynecol 2014;210:S188doi:10.1016/j.ajog.2013.10.402.

108 Murtezani A, Paçarada M, Ibraimi Z, Nevzati A, Abazi N. The impact of exercise during pregnancy on neonatal outcomes: a randomized controlled trial. J Sports Med Phys Fitness 2014;54:802-8.

109 Price BB, Amini SB, Kappeler K. Exercise in pregnancy: effect on fitness and obstetric outcomes-a randomized trial. Med Sci Sports Exerc 2012;44:2263-9. doi:10.1249/MSS.0b013e318267ad67

110 Ramirez-Velez R. Effect of recommended physical activity dose on obstetrical, neonatal and maternal metabolic outcomes in pregnant Latina women. Ann Nutr Metab 2013;63(Suppl 1):984.

111 Ronnberg AK, Ostlund I, Fadl H, Gottvall T, Nilsson K. Intervention during pregnancy to reduce excessive gestational weight gain-a randomised controlled trial. BJOG 2015;122:537-44 doi:10.1111/1471-0528.13131

112 Ramírez-Vélez R, Aguilar de Plata AC, Escudero MM. Influence of regular aerobic exercise on endothelium-dependent vasodilation and cardiorespiratory fitness in pregnant women. J Obstet Gynaecol Res 2011;37:1601-8. doi:10.1111/j.1447-0756.2011. 01582.x

113 Seneviratne SN, Jiang Y, Derraik J. Effects of antenatal exercise in overweight and obese pregnant women on maternal and perinata outcomes: a randomised controlled trial. BJOG 2016;123:588-97. doi:10.1111/1471-0528.13738

114 Arthur C, Di Corleto E, McGrath S. Daily Weight Monitoring in Pregnancy - A Randomised Controlled Trial. Aust N Z J Obstet Gynaecol 2016;56:30-3.

115 Aşcı Ö, Rathfisch G. Effect of lifestyle interventions of pregnant women on their dietary habits, lifestyle behaviors, and weight gain: a randomized controlled trial. J Health Popul Nutr 2016;35:7-16. doi:10.1186/s41043-016-0044-2

116 Bruno R, Petrella E, Bertarini V, Pedrielli G, Neri I, Facchinetti F. Adherence to a lifestyle programme in overweight/obese pregnant women and effect on gestational diabetes mellitus: a randomized controlled trial. Matern Child Nutr 2016; Epub ahead of print. doi:10.1111/mcn.12333

117 Daly N, Farren M, McKeating AEffect of an intensive medically supervised exercise program designed to improve maternal glucose control on gestational weight gain - a randomized controlled trial. Am J Obstet Gynecol 2017:(Suppl 34).

118 Garnæs KK, Mørkved S, Salvesen $\varnothing$, Moholdt T. Exercise Training and Weight Gain in Obese Pregnant Women: A Randomized Controlled Trial (ETIP Trial). PLoS Med 2016;13:e1002079. doi:10.1371/ journal.pmed.1002079

119 Kihlstrand M, Stenman B, Nilsson S, Axelsson O. Water-gymnastics reduced the intensity of back/low back pain in pregnant women. Acta Obstet Gynecol Scand 1999;78:180-5. doi:10.1080/j.16000412.1999.780302.x

120 Ko CW, Napolitano PG, Lee SP, Schulte SD, Ciol MA, Beresford SA. Physical activity, maternal metabolic measures, and the incidence of gallbladder sludge or stones during pregnancy: a randomized trial. Am J Perinatol 2014;31:39-48.

121 McCarthy EA, Walker SP, Ugoni A, Lappas M, Leong O, Shub A. Self-weighing and simple dietary advice for overweight and obese pregnant women to reduce obstetric complications without impact on quality of life: a randomised controlled trial. BJOG 2016;123:96573. doi:10.1111/1471-0528.13919

122 Parat S, Cosson E, Baptiste A. A Randomized Trial on the Effects of Perinatal Education of Overweight Pregnant Women to Prevent Childhood Overweight: the ETOIG study. Eur Soc Paed End, 2015: p1-52.

123 Peaceman AM, Kwasny MJ, Gernhofer N. MOMFIT: A randomized clinical trial of an intervention to prevent excess gestational weight gain in overweight and obese women. Am J Obstet Gynecol 2017;216:S2-3doi:10.1016/j.ajog.2016.11.003.

124 Perales M, Santos-Lozano A, Sanchis-Gomar F. Maternal Cardiac Adaptations to a Physical Exercise Program during Pregnancy. Med Sci Sports Exerc 2016;48:896-906. doi:10.1249/ MSS.0000000000000837 
125 Petrov Fieril K, Glantz A, Fagevik Olsen M. The efficacy of moderateto-vigorous resistance exercise during pregnancy: a randomized controlled trial. Acta Obstet Gynecol Scand 2015;94:35-42. doi:10.1111/aogs.12525

126 Rakhshani A, Nagarathna R, Mhaskar R, Mhaskar A, Thomas A, Gunasheela S. The effects of yoga in prevention of pregnancy complications in high-risk pregnancies: a randomized controlled trial. Prev Med 2012;55:333-40. doi:10.1016/j.ypmed.2012.07.020

127 Simmons D, Devlieger R, van Assche A. Effect of physical activity and/or healthy eating on GDM risk: The DALI Lifestyle Study. J Clin Endocrinol Metab 2017;102:903-13.

128 Smith K, Lanningham-Foster L, Welch A, Campbell C. Web-Based Behavioral Intervention Increases Maternal Exercise but Does Not Prevent Excessive Gestational Weight Gain in Previously Sedentary Women. J Phys Act Health 2016;13:587-93. doi:10.1123/ jpah.2015-0219

129 Sun Y, Zhao H. The effectiveness of lifestyle intervention in early pregnancy to prevent gestational diabetes mellitus in Chinese overweight and obese women: A quasi-experimental study. Appl Nurs Res 2016;30:125-30. doi:10.1016/j.apnr.2015.10.006

130 Tomić V, Sporiš G, Tomić J, Milanović Z, Zigmundovac-Klaić D, Pantelić S. The effect of maternal exercise during pregnancy on abnormal fetal growth. Croat Med / 2013;54:362-8. doi:10.3325/ cmj.2013.54.362

131 Toosi MA. M. The Effect of Aerobic Exercises on Maternal Outcomes: A Randomized Controlled Clinical Trial. Women's. Health Bullet 2016;3:e31337. doi:10.17795/whb-31337

132 Wang C, Wei Y, Zhang X. Effect of Regular Exercise Commenced in Early Pregnancy on the Incidence of Gestational Diabetes Mellitus in Overweight and Obese Pregnant Women: A Randomized Controlled Trial. Diabetes Care 2016;39:e163-4. doi:10.2337/dc16-1320

133 Willcox IC, Wilkinson SA, Lappas M. A mobile health intervention promoting healthy gestational weight gain for women entering pregnancy at a high body mass index: the txt4two pilot randomised controlled trial. BJOG 2017; Epub ahead of print. doi:10.1111/14710528.14552

134 Vickers AJ, Altman DG. Statistics notes: Analysing controlled trials with baseline and follow up measurements. BMJ 2001;323:1123-4. doi:10.1136/bmi.323.7321.1123

135 Riley RD, Kauser I, Bland M. Meta-analysis of randomised trials with a continuous outcome according to baseline imbalance and availability of individual participant data. Stat Med 2013;32:274766. doi:10.1002/sim. 5726

136 Gunderson EP, Abrams B. Epidemiology of gestational weight gain and body weight changes after pregnancy. Epidemiol Rev 2000;22:261-74. doi:10.1093/oxfordjournals.epirev.a018038

137 Muktabhant B, Lawrie TA, Lumbiganon P, Laopaiboon M. Diet or exercise, or both, for preventing excessive weight gain in pregnancy. Cochrane Database Syst Rev 2015;6:CD007145.

138 Tudur Smith C, Dwan K, Altman DG, Clarke M, Riley R, Williamson PR. Sharing individual participant data from clinical trials: an opinion survey regarding the establishment of a central repository. PLoS One 2014;9:e97886. doi:10.1371/journal.pone.0097886

139 Rong K, Yu K, Han X. Pre-pregnancy BMI, gestational weight gain and postpartum weight retention: a meta-analysis of observational studies. Public Health Nutr 2015:18:2172-82 doi:10.1017/ S1368980014002523

140 Knight-Agarwal CR, Williams LT, Davis D. Association of BMI and interpregnancy BMI change with birth outcomes in an Australian obstetric population: a retrospective cohort study. BMJ Open 2016;6:e010667. doi:10.1136/bmjopen-2015-010667

141 Ferraro ZM, Contador F, Tawfiq A, Adamo KB, Gaudet L. Gestational weight gain and medical outcomes of pregnancy. Obstet Med 2015;8:133-7. doi:10.1177/1753495X15591320

\section{Appendix: Supplementary materials}

